\title{
WinBUGS for Population Ecologists: Bayesian Modeling Using Markov Chain Monte Carlo Methods
}

\author{
Olivier Gimenez, Simon J. Bonner, Ruth King, Richard A. Parker, \\ Stephen P. Brooks, Lara E. Jamieson, Vladimir Grosbois, Byron J.T. Morgan \\ and Len Thomas
}

\begin{abstract}
The computer package WinBUGS is introduced. We first give a brief introduction to Bayesian theory and its implementation using Markov chain Monte Carlo (MCMC) algorithms. We then present three case studies showing how WinBUGS can be used when classical theory is difficult to implement. The first example uses data on white storks from Baden Württemberg, Germany, to demonstrate the use of mark-recapture models to estimate survival, and also how to cope with unexplained variance through random effects. Recent advances in methodology and also the WinBUGS software allow us to introduce (i) a flexible way of incorporating covariates using spline smoothing and (ii) a method to deal with missing values in covariates. The second example shows how to estimate population density while accounting for detectability, using distance sampling methods applied to a test dataset collected on a known population of wooden stakes. Finally, the third case study involves the use of state-space models of wildlife population dynamics to make inferences about density dependence in a North American duck species. Reversible Jump MCMC is used to calculate the probability of various candidate models. For all examples, data and WinBUGS code are provided.
\end{abstract}

Keywords Bayesian statistics · Density dependence $\cdot$ Distance sampling $\cdot$ External covariates $\cdot$ Hierarchical modeling $\cdot$ Line transect $\cdot$ Mark-recapture $\cdot$ Random effects $\cdot$ Reversible jump MCMC $\cdot$ Spline smoothing $\cdot$ State-space model $\cdot$ Survival estimation

\section{Introduction}

The Bayesian approach dates back to the Reverend Thomas Bayes and the 18th century. However, due to practical problems of implementing the Bayesian approach, little advance was made for over two centuries. The development of new

O. Gimenez $(\bowtie)$

CEFE/CNRS, UMR 5175, 1919, Route de Mende, 34293 Montpellier Cedex 5, France e-mail: olivier.gimenez@cefe.cnrs.fr 
methodology coupled with recent advances in computational power and the availability of flexible and reliable software have led to a great increase in the application of Bayesian methods within the last three decades, population ecology being no exception (Clark 2005; Ellison 2004; McCarthy 2007). Indeed, the application of the Bayesian theory in population ecology has been greatly facilitated by the implementation of algorithms known as Markov chain Monte Carlo (MCMC) methods (Gilks et al. 1996 and Link et al. 2002 for an introduction for ecologists) in flexible and reliable software. For example, MARK (White and Burnham 1999), one of the most popular computer programs in population ecology, now includes an MCMC option which implements a simple MCMC algorithm (White and Burnham this volume). AD Model Builder (ADMB; Fournier 2001) is a general modeling environment for fitting complex models to data, that has been used mainly in fisheries stock assessment (Maunder et al. submitted), and has an MCMC option to implement Bayesian analysis (see Maunder et al. this volume). Here we focus on the program WinBUGS (Bayesian inference Using Gibbs Sampling; Spiegelhalter et al. 2003), which implements up-to-date and powerful MCMC algorithms that are suited to a wide range of target distributions for analyzing complex models.

The paper is organized as follows. We first review the Bayesian framework and show how it can be fruitfully implemented using MCMC algorithms and program WinBUGS. We then focus on three case studies to illustrate how WinBUGS can be used to apply Bayesian methods using MCMC algorithms in population ecology. The first example deals with mark-recapture models to estimate survival probabilities and shows how to incorporate covariates with maximum flexibility. The second example shows how to estimate population density while accounting for detectability by using distance sampling methods. Finally, the third case study involves modeling count data using state-space models. We conclude with a short discussion of various possible extensions to both the methods and software that we have illustrated.

When presenting the examples, we include short illustrations in WinBUGS (code is indicated using this typeface). For all three examples, the relevant data and full WinBUGS code are given at http://eprints.st-andrews. ac.uk/archive/00000450/.

\section{The Bayesian Method Using MCMC Algorithms: Practical Implementation in WinBUGS}

Typical statistical problems involve estimating a vector of parameters, $\theta$, using the available data. The classical approach assumes that the parameters are fixed, but have unknown values to be estimated. Classical maximum likelihood estimates generally provide a point estimate of the parameter of interest. The Bayesian approach assumes that the parameters themselves have some unknown distribution. The approach is based upon the idea that the experimenter begins with some prior beliefs about the system, and then updates these beliefs on the basis of observed data. Using Bayes' Theorem, the posterior distribution of the parameters 
given the data $\pi(\theta \mid$ data $)$ has density proportional to the product of the likelihood of the data given the parameters $L(\operatorname{data} \mid \theta)$ and the prior distribution of the parameters $\pi(\theta): \pi(\theta \mid$ data $) \propto L($ data $\mid \theta) \times \pi(\theta)$. The prior distribution represents the expert's belief, before observing any data. If there is no strong prior information on the parameters, vague priors are typically specified on the parameters which represent very weak opinion concerning the model parameters. Unfortunately, in most realistic applications the posterior distribution is generally of such high dimension that little useful inference can be obtained directly. As a consequence, while the joint posterior distribution (or the corresponding marginal distributions) provide the best summaries of the parameters, point estimates and uncertainty intervals are often more interpretable. It is the process of summarizing the posterior that is the source of the computational complexity of the Bayesian approach. Estimating the summary statistics of interest (for a vector of parameters $\theta$ ) requires elimination of the other parameters. The Bayesian approach does this through integration using the MCMC algorithm. The high-dimensional integral associated with the posterior density is actually estimated using appropriate Monte Carlo integration, which consists of constructing a Markov chain with stationary distribution equal to the posterior distribution of interest. Then, once the chain has converged, realizations can be regarded as a dependent sample from this distribution. WinBUGS implements powerful ways of constructing these chains, adapting to a wide range of target (posterior) distributions and therefore allowing a large number of possible models to be fitted. Further details on Bayesian modeling using MCMC algorithms can be found in Gilks et al. (1996) and Congdon (2003, 2006). The WinBUGS software is currently freely available at http://www.mrcbsu.cam.ac.uk/bugs/.

A typical WinBUGS session proceeds as follows: the user specifies the model to run in the form of the likelihood and prior distributions for all parameters to be estimated. Data and initial values must also be provided. Following the validation of the user specification, MCMC simulations are generated such that the stationary distribution of the Markov chain is the posterior distribution of interest. Thus, this algorithm provides a sample from the posterior distribution of interest from which, it is possible to produce estimates of the posterior distributions using kernel density estimates, and summary statistics of interest such as posterior medians and credible intervals. Convergence diagnostics are also available either directly in WinBUGS or using the R packages CODA (Plummer et al. 2004) or BOA (Smith 2004). Note that we will not discuss this crucial issue here, but recommendations can be found in Kass et al. (1998). An important feature of WinBUGS is that it comes with a tutorial designed to provide new users with a step-by-step guide to running an analysis in WinBUGS. There are also a wide range of varied and detailed examples, including, for instance: logistic regression with random effects, analyses of variance with repeated measurements, meta-analyses and survival analyses with frailties. It is often useful to call WinBUGS from other programs in order to input complex sets of data and initial values, avoid specifying the parameters to be monitored in each run, post-process the results in other software, display complex graphics or perform Monte Carlo studies running WinBUGS iteratively 
in a loop. Together with data and WinBUGS codes, we give an illustration of the use of the R (Ihaka and Gentleman 1996; R Development Core Team 2007) package R2WinBUGS (Sturtz et al. 2005), as well as an illustration of how to call WinBUGS from MATLAB using the package MATBUGS (http://www.cs. ubc.ca/ murphyk/Software/MATBUGS/matbugs.html) at http://eprints.st-andrews. ac.uk/archive/00000450/. Other programs that can be used to interface to WinBUGS are listed on the WinBUGS web page given above. General and complementary introductions to WinBUGS are given in Congdon (2006) and McCarthy (2007). We now turn to the analysis of real case studies to illustrate the use of WinBUGS. Note that likelihoods and priors are implemented by defining their probability distribution based on the model parameters using the tilde $(\sim)$ symbol. This notation will be used throughout the paper.

\section{Estimating Survival Using Mark-Recapture Data}

As an illustration, we use data on the white stork Ciconia ciconia population in Baden Württemberg (Germany), consisting of 321 capture histories of individuals ringed as chicks between 1956 and 1971. From the $60 \mathrm{~s}$ to the $90 \mathrm{~s}$, all Western European stork populations were declining (Bairlein 1991). This trend is thought to be the result of reduced food availability (Schaub et al. 2005) caused by severe droughts observed in the wintering ground of storks in the Sahel region of Africa. This hypothesis has been examined in several studies (Kanyamibwa et al. 1990; Barbraud et al. 1999; Grosbois et al. in revision). In this section, we use WinBUGS and several of its features to further explore the relationship between rainfall in the Sahel and survival probabilities of the Baden Württemberg white stork population.

\subsection{Simple Models}

The standard Cormack-Jolly-Seber model (CJS, Cormack 1964; Jolly 1965; Seber 1965; Lebreton et al. 1992) considers time-dependence for the probability $\phi_{i}$ that an individual survives to occasion $i+1$ given that it is alive at time $i$, and for the probability $p_{j}$ that an individual is recaptured at time $j$. The data consist of encounter histories for each individual made of 1's corresponding to recapture or resighting and 0's otherwise. These data can be efficiently condensed in the so-called reduced m-array (e.g. Lebreton et al. 1992) which summarizes the data in the form of the number of individuals released per occasion $i$, denoted $R_{i}$, and the number of first recaptures given release at occasion $i$ at the succeeding occasions $j$, denoted $m_{i j}$. The m-array for the white stork data is provided in Table 1 .

Conditioning on the numbers released and assuming independence among cohorts, the CJS model likelihood can be written as a product of multinomial probability distributions corresponding to each row of the m-array. The probabilities corresponding to the m-array cells are complex nonlinear functions of the survival and detection probabilities. For example, the probability of the number of individuals released at occasion 3 and recaptured for the first time at occasion 5, given the number of released individuals at occasion 3 is: 
Table 1 The m-array for the White stork data set. The number of individuals released at occasion $i$ $\left(R_{i}\right)$ and the number of first recaptures at occasion $j$, given release at occasion $i\left(m_{i j}\right)$ are provided. For example, 38 birds were released in 1969 among which, 22 were first recaptured in 1970, and $16(=38-22)$ were never observed again

\begin{tabular}{|c|c|c|c|c|c|c|c|c|c|c|c|c|c|c|c|c|c|}
\hline \multirow{2}{*}{$\begin{array}{l}\text { Year of } \\
\text { release (19-) }\end{array}$} & \multirow{2}{*}{$\begin{array}{l}\text { Number } \\
\text { released }\end{array}$} & \multicolumn{16}{|c|}{ Year of first recapture (19-) } \\
\hline & & 57 & 58 & 59 & 60 & 61 & 62 & 63 & 64 & 65 & 66 & 67 & 68 & 69 & 70 & 71 & 72 \\
\hline 56 & 26 & 19 & 2 & 0 & 0 & 0 & 0 & 0 & 0 & 0 & 0 & 0 & 0 & 0 & 0 & 0 & 0 \\
\hline 57 & 50 & 0 & 33 & 3 & 0 & 0 & 0 & 0 & 0 & 0 & 0 & 0 & 0 & 0 & 0 & 0 & 0 \\
\hline 58 & 53 & 0 & 0 & 35 & 4 & 0 & 0 & 0 & 0 & 0 & 0 & 0 & 0 & 0 & 0 & 0 & 0 \\
\hline 59 & 69 & 0 & 0 & 0 & 42 & 1 & 0 & 0 & 0 & 0 & 0 & 0 & 0 & 0 & 0 & 0 & . \\
\hline 60 & 73 & 0 & 0 & 0 & 0 & 42 & 1 & 0 & 0 & 0 & 0 & 0 & 0 & 0 & 0 & 0 & 0 \\
\hline 61 & 71 & 0 & 0 & 0 & 0 & 0 & 32 & 2 & 1 & 0 & 0 & 0 & 0 & 0 & 0 & 0 & 0 \\
\hline 62 & 64 & 0 & 0 & 0 & 0 & 0 & 0 & 46 & 2 & 0 & 0 & 0 & 0 & 0 & 0 & 0 & 0 \\
\hline 63 & 64 & 0 & 0 & 0 & 0 & 0 & 0 & 0 & 33 & 3 & 0 & 0 & 0 & 0 & 0 & 0 & 0 \\
\hline 64 & 66 & 0 & 0 & 0 & 0 & 0 & 0 & 0 & 0 & 44 & 2 & 0 & 0 & 0 & 0 & 0 & 0 \\
\hline 65 & 55 & 0 & 0 & 0 & 0 & 0 & 0 & 0 & 0 & 0 & 43 & 1 & 0 & 0 & 1 & 0 & 0 \\
\hline 66 & 60 & 0 & 0 & 0 & 0 & 0 & 0 & 0 & 0 & 0 & 0 & 34 & 1 & 0 & 0 & 0 & 0 \\
\hline 67 & 53 & 0 & 0 & 0 & 0 & 0 & 0 & 0 & 0 & 0 & 0 & 0 & 36 & 1 & 0 & 0 & 0 \\
\hline 68 & 51 & 0 & 0 & 0 & 0 & 0 & 0 & 0 & 0 & 0 & 0 & 0 & 0 & 27 & 2 & 0 & 0 \\
\hline 69 & 38 & 0 & 0 & 0 & 0 & 0 & 0 & 0 & 0 & 0 & 0 & 0 & 0 & 0 & 22 & 0 & 0 \\
\hline 70 & 33 & 0 & 0 & 0 & 0 & 0 & 0 & 0 & 0 & 0 & 0 & 0 & 0 & 0 & 0 & 15 & 1 \\
\hline 71 & 23 & 0 & 0 & 0 & 0 & 0 & 0 & 0 & 0 & 0 & 0 & 0 & 0 & 0 & 0 & 0 & 15 \\
\hline
\end{tabular}

$$
\phi_{3}\left(1-p_{4}\right) \phi_{4} p_{5}
$$

For further details of fitting the CJS model in a Bayesian framework, see Brooks et al. (2000). We start with a simple mark-recapture model, a simplification of the CJS model where, based on the conclusions of previous studies (Kanyamibwa et al. 1990; Grosbois et al. in revision), the recapture probabilities are considered constant over time.

\subsubsection{Defining Priors}

We define priors for the survival probabilities and the recapture probability as Beta distributions with parameters 1 and 1 (equivalently uniform distributions between 0 and 1). Within WinBUGS, this is specified as:

for (i in 1:ni) \{phi [i] abeta $(1,1)\}$

$\mathrm{p} \sim$ dbeta $(1,1)$

where $\mathrm{ni}$ is the number of occasions of release in the study.

\subsubsection{Constructing the Likelihood}

The likelihood is defined as a product of multinomial distributions using the function dmulti :

for (i in $1: n i)\{m[i, 1:(n j+1)] \sim \operatorname{dmulti(q[i,],r[i])\} }$

where the $m$ object is the m-array matrix of data (augmented by the number of individuals never seen again after release in the last column), $n j$ is the number of 
recapture occasions within the study, $r$ is the vector of released individuals and $q$ is a matrix of the m-array cells probabilities. The $q$ matrix and $r$ vector are calculated in the WinBUGS code.

\subsubsection{Results}

The posterior medians of the survival probabilities are displayed in Fig. 1a, along with their posterior $95 \%$ credible intervals.

To check that the temporal variations in the survival are worth considering, we also consider a compromise approach in which survival is taken as constant over time. Starting from the code of the previous model, one way to proceed would be to consider one scalar parameter for the survival, specify the prior distribution as for the detection probability and modify the likelihood accordingly. A neat trick which avoids modifying the likelihood part of the code, is to define a single dummy variable with a Beta prior and then set all survival probabilities equal to that variable:

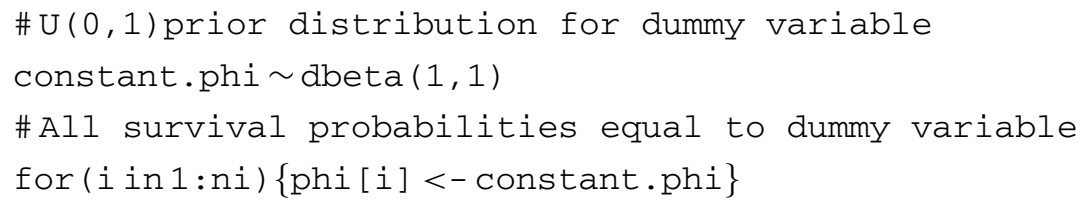

\subsubsection{DIC for Model Selection}

As a preliminary model selection technique, we use the Deviance Information Criterion (DIC; Spiegelhalter et al. 2002). One interpretation of the DIC is as a Bayesian counterpart to the AIC for model selection. Essentially, the DIC is a diagnostic that balances the requirements of model fit and low complexity. Typically, as models get more complex by the addition of extra parameters, their fit improves. The DIC diagnostic therefore penalizes additional parameters so that a parsimonious model is chosen, and the smaller the DIC value, the better the compromise is. One advantage is that the DIC can be calculated directly in WinBUGS from the chains produced by an MCMC run. However, the DIC statistic is in its infancy and is controversial (see the discussion papers following Spiegelhalter et al. 2002 and Celeux et al. 2006). Here we consider the DIC as a preliminary tool for comparing competing models, and we will discuss a more rigorous approach later, in the form of posterior model probabilities.

Examining the DIC values in Table 2, we see that the time-dependent model appears to outperform the constant model, and hence is better supported by the data. This suggests that dependence upon time is needed to explain variations in the survival probabilities. To better understand these findings, we will consider in the next section environmental covariates as possibly explaining time variation in the survival probabilities. 
(a)

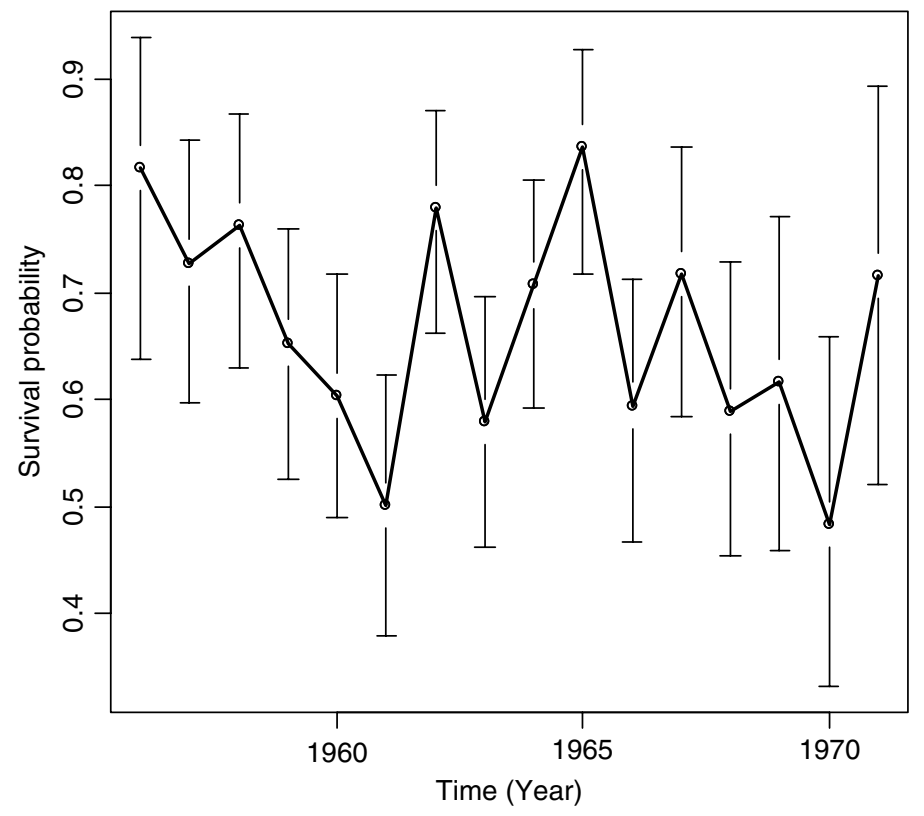

(b)

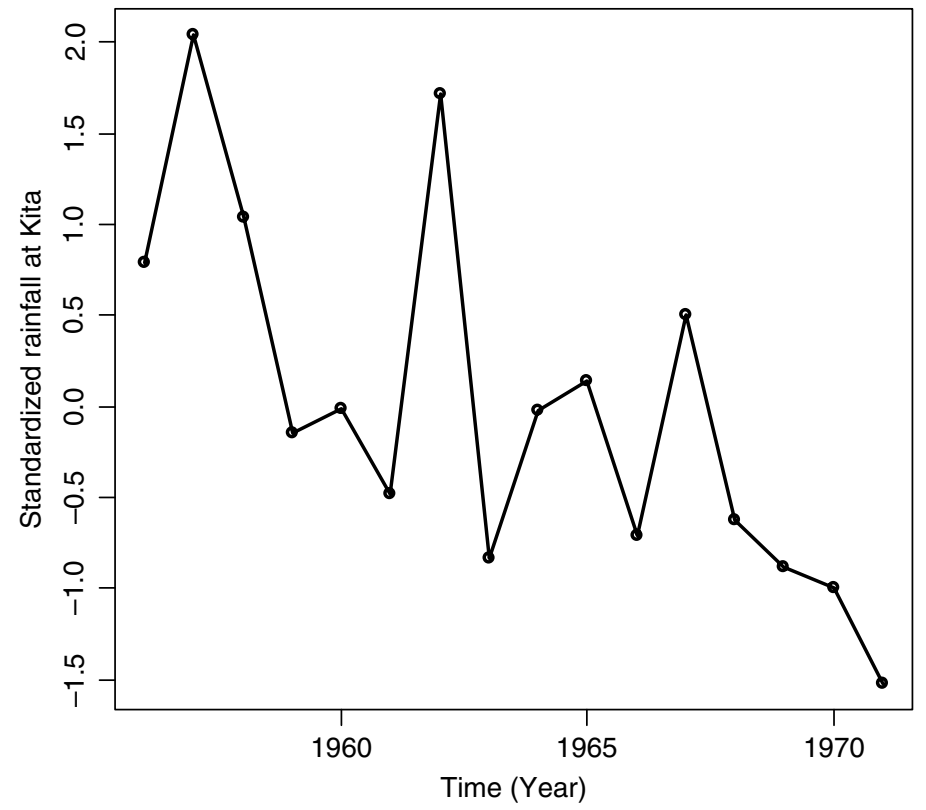

Fig. 1 (a) White stork survival estimates from model with time-dependent survival probabilities and constant detection probabilities; vertical bars represent 95\% pointwise credible intervals; (b) rainfall time series at meteorological station Kita in the Sahel 
Table 2 Models fitted to the white stork data. DIC is the deviance information criterion and $\mathrm{pD}$ is the number of effective parameters. See text for details

\begin{tabular}{llr}
\hline Model & DIC & $\mathrm{pD}$ \\
\hline Constant survival probabilities & 174.3 & 1.9 \\
Time-dependent survival probabilities & 166.0 & 16.3 \\
Covariate-dependent survival probabilities & 159.4 & 3.1 \\
Covariate-dependent as well as random-effect & 161.0 & 10.6 \\
$\quad$ survival probabilities & & \\
Nonparametric survival probabilities & 158.1 & 7.4 \\
\hline
\end{tabular}

\subsection{Incorporating Linear Effects of Covariates}

We now turn to the incorporation of covariates in the CJS model (North and Morgan 1979; Pollock et al. 1984; Clobert and Lebreton 1985; Lebreton et al. 1992; see Pollock 2002 for a review). As we mentioned earlier, the variation in white storks survival is likely to be related to rainfall variations. As expected, it can be seen that the variations in the survival estimates (Fig. 1a) are correlated to Sahel rainfall variations (Fig. 1b). According to Williams et al. (2002, p. 373), we therefore consider a model including a linear effect of the rainfall covariate on the logit scale:

$$
\operatorname{logit}\left(\phi_{i}\right)=\log \left(\frac{\phi_{i}}{1-\phi_{i}}\right)=\beta_{1}+\beta_{2} x_{i},
$$

where $x_{i}$ is the value of the covariate between occasions $i$ and $i+1$, and the $\beta$ 's are regression parameters to be estimated. We use normal distributions with mean 0 and large variance $\left(10^{6}\right)$ as vague prior distributions for those parameters. The rainfall measurements are standardized to improve mixing within the Markov chain. Note that the standardization can be implemented in WinBUGS:

for $(i \operatorname{in} 1: \operatorname{ni})\{\operatorname{cov}[i]<-(\operatorname{cov}[i]-\operatorname{mean}(\operatorname{cov}[])) / \operatorname{sd}(\operatorname{cov}[])\}$

where cov [i] denotes the covariate value in year $i$.

The code provided in the previous section is amended as follows:

for (i in $1: n i)\{\operatorname{logit}(\operatorname{phi}[i])<-\operatorname{beta}[1]+\operatorname{beta}[2] * \operatorname{cov}[i]\}$

for $(j \operatorname{in} 1: 2)\{\operatorname{beta}[j] \sim \operatorname{dnorm}(0,1.0 \mathrm{E}-6)\}$

Note that in WinBUGS, normal distributions are described in terms of a mean and precision, where precision $=1 /$ variance. As a consequence, a variance of 1,000,000 corresponds to a precision of 0.000001 . In addition, we note that this model makes the strong assumption that variation in the survival probabilities is explained by the covariate. This can be relaxed by the inclusion of additional random effects.

\subsection{Incorporating Random Effects}

We consider two models with random effects in this section, both addressing two different questions. Note that incorporating random effects is also a way to share 
information among parameters, particularly improving estimates for years where there is little information in the data (e.g. Harley et al. 2004).

First, specifying constant survival probabilities can be too restrictive to capture sources of temporal variability, while estimating as many parameters as time intervals may be too costly to assess specific time trends (Burnham and White 2002; Royle and Link 2002). We consider a compromise model where time is treated as a random effect, $\varepsilon$, with a normal distribution with mean 0 and variance $\sigma^{2}$. We therefore estimate the mean logit survival probability, say $\mu$, and the temporal process variance in survival probability $\sigma^{2}$ (Gould and Nichols 1998; Burnham and White 2002):

$$
\operatorname{logit}\left(\phi_{i}\right)=\mu+\varepsilon_{i}
$$

Considering random effects raises the problem of calculating the likelihood, which is obtained by integrating over the random effect $\varepsilon$. This is, indeed, a problem involving a high-dimensional integral that could be handled by using approximations (Chavez-Demoulin 1999), circumvented by resorting to asymptotic arguments (Gould and Nichols 1998; Burnham and White 2002), or numerical integration (e.g. importance sampling: Skaug and Fournier 2006 or Gaussian quadrature: Wintrebert et al. 2005). By contrast, the Bayesian approach provides an exact solution to this problem (Brooks et al. 2000, 2002, note that both references contain WinBUGS code) and WinBUGS offers a powerful and flexible alternative to standard software such as MARK (White and Burnham 1999) or M-SURGE (Choquet et al. 2005).

The specification of the model for the survival probabilities was as follows:

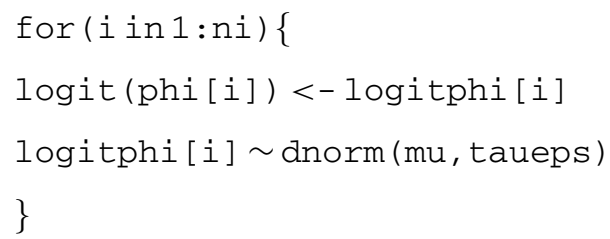

We consider an inverse-gamma distribution with parameters 0.01 and 0.01 and a normal distribution with mean 0 and large variance (100) as vague prior distributions for taueps and respectively mu:

taueps $\sim \operatorname{dgamma}(0.01,0.01)$

$\mathrm{mu} \sim \operatorname{dnorm}(0,0.01)$

Note that a gamma distribution for the precision is equivalent to an inverse-gamma distribution for the variance. In this case, these are typical specifications of vague priors (see also Lambert et al. 2005; van Dongen 2006; Gelman 2006). The posterior distribution of the variance can easily be obtained by monitoring the quantity sigma2eps defined as:

sigma2eps $<-1 /$ taueps

Second, the inclusion of random effects allows there to be additional variability within the survival rates that can be attributed to natural variability, or temporal 
variability not explained by the covariates within the study. This is a simple extension of the above covariate model. In particular, we specify an additional random effect term denoted by $\varepsilon$, which has a normal distribution with mean 0 and variance $\sigma^{2}$. In particular we model the survival rate to be of the form:

$$
\operatorname{logit}\left(\phi_{i}\right)=\beta_{1}+\beta_{2} x_{i}+\varepsilon_{i}
$$

Then, the parameters to be estimated are the regression coefficients ( $\beta$ 's) and the random effect variance parameter $\sigma^{2}$. In a particular application, Barry et al. (2003) noticed that omitting the random effect can lead to overestimation of the significance of the covariate on survival. To include these additional random effects, the code is modified as follows:

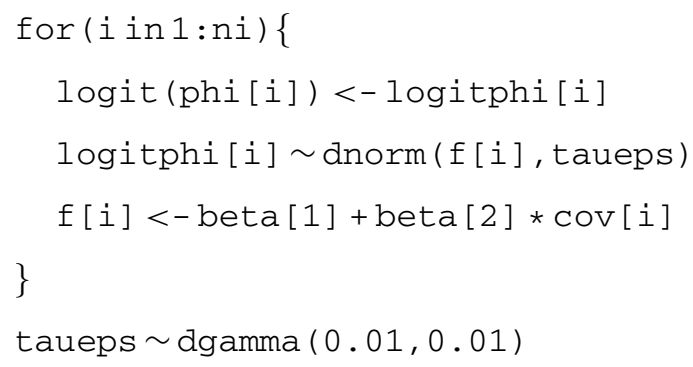

In our model, slope estimates produced using Eqs. (2) and (4) to model survival are very close to each other: posterior medians for the slope $\beta_{2}$ were 0.36 in both cases with $95 \%$ credible intervals $[0.14 ; 0.58]$ and $[0.20 ; 0.55]$ (see Fig. 2). This may indicate that the random effect was not needed in the model, as the estimates tend to confirm (the distribution of $\sigma^{2}$ places all of its mass near 0 with posterior median 0.04 and $95 \%$ credible interval $[0.01 ; 0.22])$, and indicated by the preliminary DIC analysis (see Table 2).

A formal way of testing the null hypothesis $\sigma^{2}=0$ will be discussed later. In both cases, the effect of rainfall is positive, indicating that the more it rained in the Sahel zone, the better storks survived.

\subsection{Nonparametric Modeling}

There is another strong assumption made in Eq. (2), namely that the effect of the covariate on the survival probability is linear on the logit scale. However, nonlinear relationships involving the impact of environmental factors on population dynamics may occur (Mysterud et al. 2001). More flexible models for the survival probability are therefore needed. Gimenez et al. (2006a; see also Gimenez and Barbraud this volume and Gimenez et al. 2006b for a similar approach applied to individual covariates) have recently proposed a method in which the shape of the relationship is determined by the data without making any prior assumption regarding its form, by using penalized splines (P-splines; Ruppert et al. 2003). Here, 
(a)

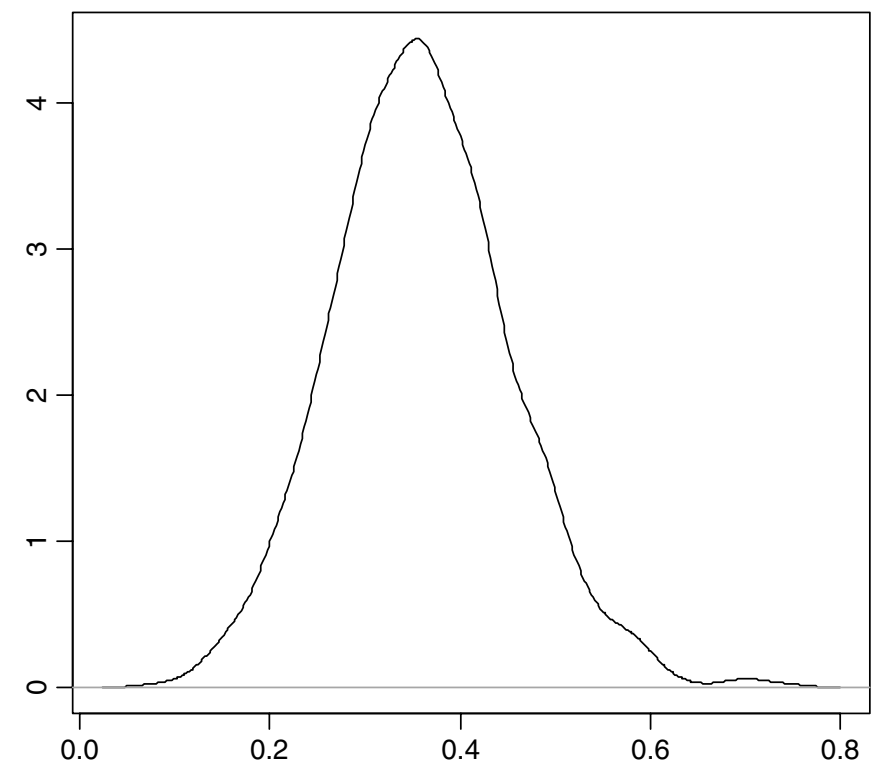

(b)

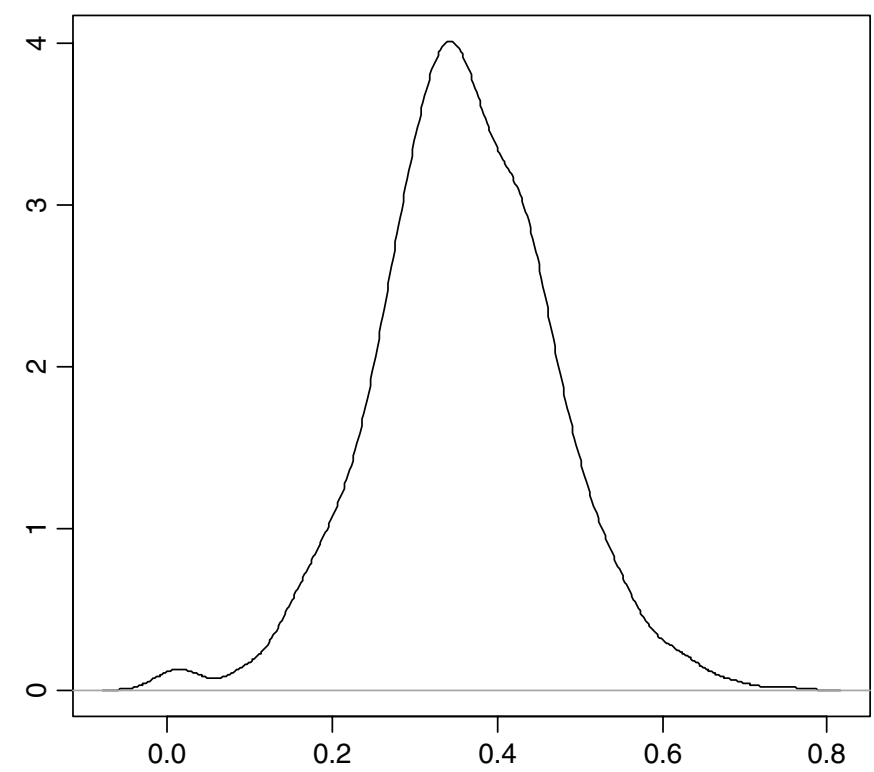

Fig. 2 Posterior distributions of the regression parameter $\beta_{2}$ corresponding to the rainfall effect on annual white stork survival (a) without and (b) with a random effect 
we give details of how to implement their approach in WinBUGS. We consider the following regression model for the survival probability $\phi_{i}$ :

$$
\operatorname{logit}\left(\phi_{i}\right)=f\left(x_{i}\right)+\varepsilon_{i},
$$

where $x_{i}$ is the value of the covariate between occasions $i$ and $i+1, f$ is a smooth function and $\varepsilon_{i}$ are i.i.d. random effects $N\left(0, \sigma_{\varepsilon}^{2}\right)$. The function $f$ specifies a nonparametric flexible relationship between the survival probability and the covariate that allows nonlinear environmental trends to be detected. Following Gimenez et al. (2006a), we use a truncated polynomial basis to describe $f$ :

$$
f(x)=\beta_{0}+\beta_{1} x+\ldots+\beta_{p} x^{p}+\sum_{k=1}^{K} b_{k}\left(x-\kappa_{k}\right)_{+}^{p},
$$

where $x$ is the covariate, and $\beta_{0}, \beta_{1}, \ldots, \beta_{p}, b_{1}, \ldots, b_{K}$ are regression coefficients to be estimated, $P \geq 1$ is the degree of the spline, $(u)_{+}^{p}=u^{p}$ if $u \geq 0$ and 0 otherwise, and $\kappa_{1}<\kappa_{2}<\ldots<\kappa_{K}$ are fixed knots. We use $K=\min \left(\frac{1}{4} I, 35\right)$ knots to ensure the desired flexibility, and let $k_{k}$ be the sample quantile of $x$ 's corresponding to probability $\frac{k}{K+1}$. Those quantities are calculated outside WinBUGS in program $\mathrm{R}$. In particular, we model the relationships using a linear $(P=1) \mathrm{P}$-spline with $K=4$ knots implemented through the WinBUGS constants degree and nknots. To avoid overfitting, we penalize the $b$ 's by assuming that the coefficients of $\left(x-\kappa_{k}\right)_{+}^{P}$ are normally distributed random variables with mean 0 and variance $\sigma_{b}^{2}$ to be estimated. This is the reason why this approach is referred to as penalized splines (Ruppert et al. 2003). Note that an alternative to P-splines called adaptive splines (Biller 2000) is considered in the mark-recapture context by Bonner et al. (this volume). The penalization is achieved by specifying:

for ( $\mathrm{kin} 1: \operatorname{nknots})\{\mathrm{b}[\mathrm{k}] \sim \operatorname{dnorm}(0$, taub $)\}$

where the variance parameter is given an inverse-gamma distribution (i.e. the precision has a gamma distribution):

taub $\sim$ dgamma $(0.001,0.001)$

A by-product of this approach is that the amount of smoothing is automatically calculated as $\sigma_{b}^{2} / \sigma_{\varepsilon}^{2}$. To implement the P-splines model in WinBUGS, it is convenient to express it as a Generalized Linear Mixed Model (GLMM), as shown by Crainiceanu et al. (2005). If $X$ is the matrix with $i$ th row $X_{i}=\left(1, x_{i}, \ldots, x_{i}^{P}\right)^{T}$ and $Z$ the matrix with the $i$ th row $Z_{i}=\left(\left(x_{i}-\kappa_{1}\right)_{+}^{P}, \ldots,\left(x_{i}-\kappa_{K}\right)_{+}^{P}\right)^{T}$, then an equivalent model representation of Eqs. (5) and (6) in the form of a GLMM is given by Gimenez et al. (2006a):

$$
\operatorname{logit}(\phi)=X \beta+Z b+\varepsilon, \operatorname{cov}\left(\begin{array}{l}
b \\
\varepsilon
\end{array}\right)=\left(\begin{array}{cc}
\sigma_{b}^{2} I & 0 \\
0 & \sigma_{\varepsilon}^{2} I
\end{array}\right)
$$


We are now able to implement the P-splines model in WinBUGS. To code Eq. (7), we used:

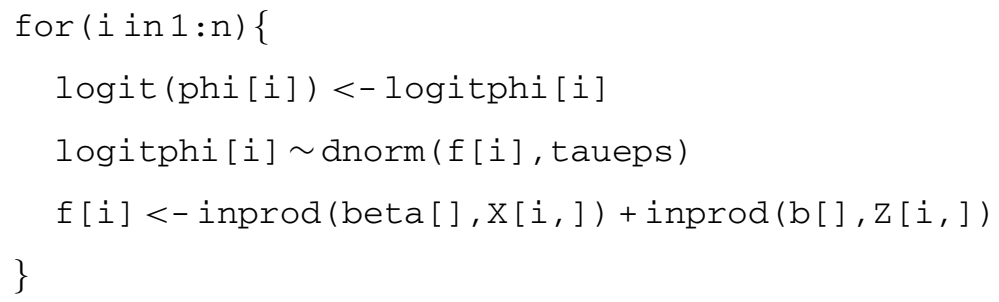

The first statement corresponds exactly to Eq. (6), the second implements the random effects distribution and the last one specifies the structure of the mean logit survival, where the function inprod denotes the inner product of two vectors. The first part of the last statement contains the fixed effect of Eq. (7), where beta[] is the vector $\beta=\left(\beta_{0}, \beta_{1}, \beta_{2}\right), \mathrm{X}[\mathrm{i}$,$] is X_{i}$ and inprod (beta [], $\mathrm{X}[\mathrm{i}$,$] ) is the$ polynomial part. The second part of the last statement contains the random effects, where $\mathrm{b}\left[\mathrm{]}\right.$ is the vector $b=\left(b_{1}, b_{2}, b_{3}, b_{4}\right), \mathrm{z}\left[\mathrm{i}, \mathrm{]}\right.$ is $Z_{i}$ and $\operatorname{inprod}(\mathrm{b}[\mathrm{]}, \mathrm{z}[\mathrm{i}, \mathrm{]})$ is the truncated polynomial part of the regression in Eq. (7).

We then obtain matrices $X$ and $Z$ directly in WinBUGS, although this step could be done in program $\mathrm{R}$ for example. Matrix $X$ is obtained as:

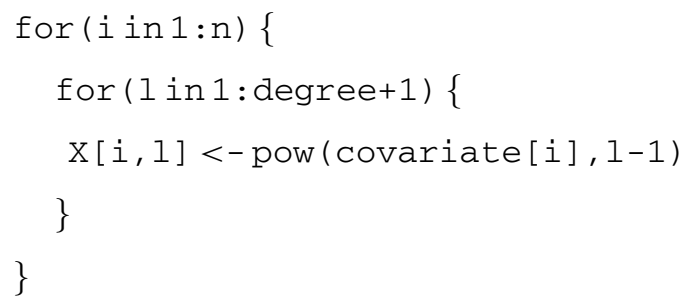

where pow is the power function, and pow $(\mathrm{a}, \mathrm{b})$ is $a^{b}$. Matrix $Z$ is obtained using: for $(i \operatorname{in} 1: n)\{$

where the function step is used to obtain the truncation, where step $(x)$ is 1 if $x$ is positive and 0 otherwise, so that $\mathrm{Z}[\mathrm{i}, \mathrm{k}]$ is positive only for $x_{i}>\kappa_{k}$. For further details see Crainiceanu et al. (2005) and Gimenez et al. (2006a). With the possibility of fitting nonparametric models, one is obviously interested in testing for the presence of nonlinearities in the survival probability regression. We address this question by using the DIC and also using visual comparison for comparing the model with a linear effect of rainfall as well as a random effect (see previous section) 


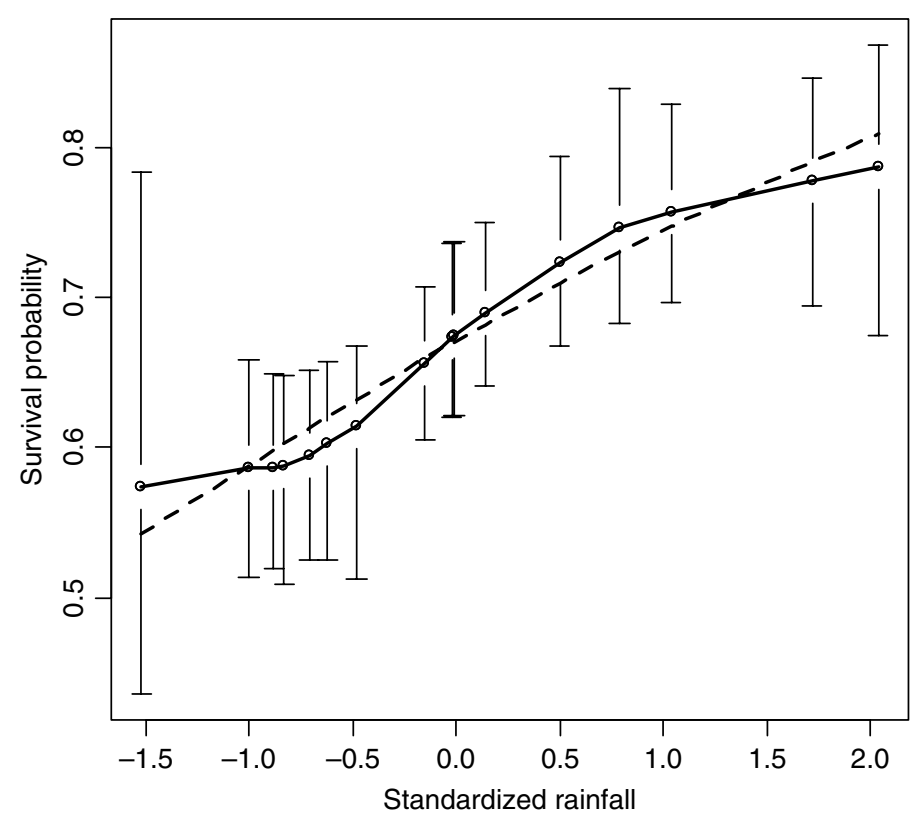

Fig. 3 Annual variations in white stork survival as a function of the standardized rainfall using a nonparametric model. Medians (solid line) with $95 \%$ pointwise credible intervals (vertical solid lines) are shown, along with the estimated linear effect (dotted line)

to its nonparametric counterpart. Figure 3 shows that the relationship between rainfall in Sahel and white stork survival can be taken as linear. This is confirmed by DIC values that are similar for these two models (Table 2). Although we have clues for linearity in this example, the issue of formally detecting nonlinearity deserves further investigation.

\subsection{Dealing with Missing Data}

Bayesian modeling via MCMC also provides a simple method for handling data with missing covariate values. Missing data might occur in capture-recapture studies if the value of an environmental covariate is not recorded on all occasions or if an individual covariate changes over time and can only be observed on the occasions when the specific animal is captured (Bonner and Schwarz 2006). Essentially, a completed data set is generated on each iteration of the MCMC algorithm by specifying an underlying model for the covariate and imputing the missing values of the covariate using the current values of the parameters, and then the completed data set is used to update the parameter values. The result is a sample from the joint posterior distribution of both the parameters and the missing data values, which can be used in Bayesian inference. We illustrate the issue of dealing 
with missing data by estimating the effect of rainfall in the Sahel on the survival of the white storks in Baden Württemberg after deleting the covariate for several years.

As with the model incorporating random effects, computing the value of the likelihood for a given set of parameter values requires integration with respect to the missing covariates. This can be a complicated numerical problem, especially if several values are missing, and is an obstruction to computing maximum likelihood estimates and their standard errors. From a Bayesian perspective, we view the missing covariates as random variables to which we can assign a probability distribution, just like the model parameters. We define a prior distribution for the missing covariate values and then compute the posterior distribution of both the parameters and the missing values conditional on the observed data. The likelihood function used in the analysis is exactly the same function used when all covariate values are observed, and if MCMC is used to obtain a sample from the posterior distribution then no additional integration is required. Instead, a sample of probable values for the missing covariates is generated by sampling new values on each iteration of the MCMC algorithm in exactly the same way that model parameters are sampled. The prior distribution of the missing covariate can be chosen to capture prior beliefs about the values of the missing covariates and their relation to the rest of the data. A simple, vague prior for the rainfall in year $i, x_{\mathrm{i}}$, is the normal distribution with mean 0 and large variance $x_{i} \sim N\left(0,10^{6}\right)$. This prior distributes its mass evenly over a very wide range of values and assumes independence of the rainfall across the years of the study. Alternative prior distributions will relate the values of the covariates to each other or to other quantities. Here we use a hierarchical prior that models the change in the covariate over time as $x_{i} \sim N\left(x_{i-1}+\mu, \sigma_{x}^{2}\right)$. This asserts that the change in the covariate between adjacent years is normally distributed with the same mean and variance for all years. Information from the observed covariate values will then be used in determining the posterior mean and variance of the missing values. To complete the prior distribution we must also specify the marginal distribution of the first covariate value, $x_{1}$, and the distributions for the hyperparameters, $\mu$ and $\sigma_{x}^{2}$. Here we use the vague prior $x_{1} \sim N\left(0,10^{6}\right)$ for marginal prior of the first covariate, and the standard vague priors for a normal mean and variance: $\mu \sim N\left(0,10^{6}\right)$ and $\sigma_{x}^{2} \sim \Gamma^{-1}(0.01,0.01)$. Alternate prior specifications for the covariate values include autoregressive models, regression of the covariate against time, or relation of the covariate to other variables that might have been recorded. Adapting the WinBUGS code to account for the missing covariate values requires two simple changes: (i) adding the prior distribution for the covariates, and (ii) modifying the input data. The WinBUGS code for the hierarchical prior is:

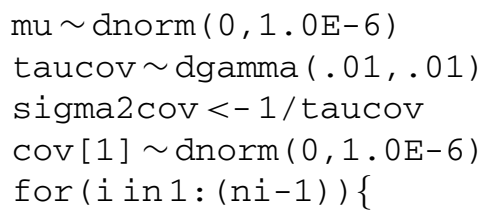




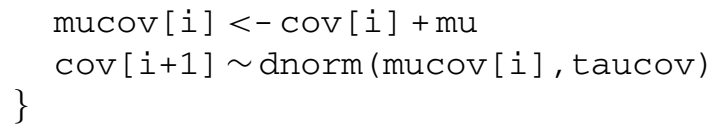

The first three lines of code define the hyperpriors for the hyperparameters ( $\mu$ is $\mathrm{mu}$ and $\sigma_{x}^{2}$ is sigma $2 \mathrm{cov}$ ). The 4th line defines the marginal prior for $x_{1}$ and the for loop defines the distribution of each of the remaining covariate values conditional on the previous value $\left(x_{\mathrm{i}}\right.$ is $\left.\mathrm{COV}[i]\right)$. Missing values in the input data are specified by replacing the observed value with ' $N A$ '. Suppose that the rainfall is observed in all years except year 15; the input vector for the covariate is:

$$
\begin{aligned}
\mathrm{COV}= & \mathrm{c}(.79,2.04,1.04,-.15,-.01,-.48,1.72,-.83,-.02, .14, \\
& -.71, .50,-.62,-.88, \mathrm{NA},-1.52)
\end{aligned}
$$

Given this data and the model above, WinBUGS will simulate values for the hyperparameters and the missing rainfall observation for year 15 on each MCMC iteration and produce posterior summaries for these quantities, exactly as it does for the other model parameters. Posterior summary statistics for a single run are shown in Table 3.

Estimates of the survival probabilities are almost exactly identical to the estimates produced from the full data; differences in the posterior means and standard

Table 3 Summary statistics for the posterior distributions of the model fitted to the white stork data with survival as a function of rainfall: no missing value, missing value in 1 year (15), missing value in 5 years $(5,6,11,12,13)$. Reported statistics are the estimated mean, standard deviation (SD), and the $95 \%$ credible interval $[\mathrm{CI}]$

\begin{tabular}{llll}
\hline & $\begin{array}{l}\text { No missing value } \\
\text { Parameter }\end{array}$ & $\begin{array}{l}\text { 1 missing value } \\
\text { Post. mean }(\mathrm{SD})[\mathrm{CI}]\end{array}$ & \multicolumn{1}{l}{ 5 missing values } \\
Post. mean $(\mathrm{SD})[\mathrm{CI}]$
\end{tabular}


deviations are the magnitude as the MCMC error. There is a very slight increase in the posterior variability of the regression coefficients, $\beta_{1}$ and $\beta_{2}$, however the lower bound of the $95 \%$ credible interval for $\beta_{2}$ is still well above 0 indicating a clear positive link between rainfall and the storks' survival. The estimated mean change in rainfall is -.15 with $95 \%$ credible interval $(-.85, .56)$ which suggests that there is no consistent trend over time. The standard deviation of the change in rainfall is relatively large which indicates that there is little association between rainfall in adjacent years. Because of this, the posterior distribution for rainfall in the missing year is uninformative about the true value.

When 5 missing values are generated, there are only minor differences in the posterior distribution of the survival probabilities with small increases in the standard deviation apparent for the years with the covariate deleted (Table 3 ). This is not surprising because the capture probabilities are very high so that most information about the survival probabilities is derived from a direct comparison of the capture histories rather than the regression on the covariate. There is, however, significant change in the inference for the regression coefficients. The posterior mean of the slope, $\beta_{2}$, is closer to 0 in Table 3, though whether the mean is increased or decreased depends on which years are missing the covariate. More importantly, the posterior standard deviation is increased from 0.11 to 0.13 and the $95 \%$ credible interval contains 0 which brings the effect of rainfall on survival into doubt.

To close this section, we note that we have only considered rainfall at a single meteorological station in the Sahel region. However, rainfall measurements at other stations are available, therefore possibly providing a better spatial representation of the white storks' wintering area. The question is then to determine which combination of the stations best explains the variation in survival. If we have 10 stations, we need to perform model selection among a set of $1024\left(2^{10}\right)$ possible candidates, which would be intractable using classical model selection criteria such as AIC, BIC or DIC. Fortunately, an alternative method can be used that allows model selection among a large set of candidate models. An example is given later in the section dealing with state-space modeling of count data, and we have made available the WinBUGS code to implement this approach on the stork dataset.

\section{Estimating Abundance and Population Density Using Line-Transect Data}

Line transect surveys are widely used to estimate the density and/or abundance of wildlife populations. The methods, which are a special case of a general approach called distance sampling, are described in detail, from a classical perspective, by Buckland et al. (2001, 2004a). Observers walk along a set of randomly located transect lines recording the perpendicular distance to all detected objects of interest (usually animals) within some detected with some perpendicular truncation distance $w$. Not all objects within distance $w$ are assumed to be detected; rather a (semi-) parametric model is specified for the probability of detecting an object given it is at perpendicular distance $y$ from the transect line. Under various assumptions (detailed 
in Buckland et al. 2001), it is then possible to derive the probability density function $f(y)$ of observed distances. This can be fitted to the observed distance data using maximum likelihood methods, and used to correct for the objects missed during the survey. The standard formula for estimating object density, D, is (Buckland et al. 2001):

$$
\hat{D}=\frac{n \hat{f}(0)}{2 L}
$$

where $n$ is the number of objects detected, $L$ is the total length of the transect lines and $\hat{f}(0)$ is the estimated probability density function of observed distances evaluated at zero distance.

As an illustration, we consider a line transect study where a known number of wooden stakes are placed in a sagebrush meadow east of Logan, Utah (Buckland et al. 2001). The true density of stakes is known to be 37.5 stakes/hectare. Eleven different graduate students walked a 1,000 m long transect through the study area independently of one another and recorded perpendicular sighting distances to stakes. One student's data are given in Table 4.

These data consist of 68 observations with a truncation width, $w$, of $20 \mathrm{~m}$. The same data set is analyzed by Karunamuni and Quinn (1995) who propose a Bayesian approach for line transect sampling. For the sake of simplicity, we make the same assumptions as Karunamuni and Quinn (1995), i.e. we assume that the probability density function $f(y)$ for the detection distances is half-normal and that the data are neither truncated nor grouped into distance intervals (see Buckland et al. 2001 for more on the latter). Thus,

$$
f(y)=\sqrt{2 / \pi \sigma^{2}} \exp \left(-y^{2} / 2 \sigma^{2}\right)=c \sqrt{\lambda} \exp \left(-\lambda y^{2} / 2\right), y>0
$$

where $c=\sqrt{2 / \pi}$ and $\lambda=1 / \sigma^{2}$. Given $n$ detection values, $y_{1}, \ldots y_{n}$, the maximum likelihood estimator of $f(0)$ is then given by:

$$
\hat{f}(0)=\sqrt{\frac{2 n}{\pi \sum y_{i}^{2}}}=c\left(\frac{T}{n}\right)^{-\frac{1}{2}}
$$

where $T=\sum y_{i}^{2}$. The maximum likelihood estimator of the density is given by Eq. (8), above.

Table 4 Sequence of perpendicular distance values for the Stakes line transect example (in meters)

\begin{tabular}{rrrrrrrrrr}
\hline 2.02 & 0.45 & 10.40 & 3.61 & 0.92 & 1.00 & 3.40 & 2.90 & 8.16 & 6.47 \\
5.66 & 2.95 & 3.96 & 0.09 & 11.82 & 14.23 & 2.44 & 1.61 & 31.31 & 6.50 \\
8.27 & 4.85 & 1.47 & 18.60 & 0.41 & 0.40 & 0.20 & 11.59 & 3.17 & 7.10 \\
10.71 & 3.86 & 6.05 & 6.42 & 3.79 & 15.24 & 3.47 & 3.05 & 7.93 & 18.15 \\
10.05 & 4.41 & 1.27 & 13.72 & 6.25 & 3.59 & 9.04 & 7.68 & 4.89 & 9.10 \\
3.25 & 8.49 & 6.08 & 0.40 & 9.33 & 0.53 & 1.23 & 1.67 & 4.53 & 3.12 \\
& 3.05 & 6.60 & 4.40 & 4.97 & 3.17 & 7.67 & 18.16 & 4.08 & \\
\hline
\end{tabular}


Adopting a Gamma prior distribution with parameters $a$ and $b$ for $\lambda$, Karunamuni and Quinn (1995) show that the posterior distribution of $\lambda$ is also a Gamma distribution with parameters $a+\frac{n}{2}$ and $\left(\frac{1}{b}+\frac{T}{2}\right)^{-1}$. Although classical Monte Carlo simulations could be used to simulate observations from the posterior distribution of $\lambda$, we use WinBUGS to draw random samples using MCMC techniques. This is motivated by generalizations to other probability density functions for the detection distances as well as spatial modeling for which explicit posterior distributions are difficult to obtain. We use the so-called "zeros trick" to implement the half-normal likelihood distribution because it is not included in the list of standard WinBUGS sampling distributions. This method consists of considering an observed data set made of 0's distributed as a Poisson distribution with parameter $\phi$ so that the associated likelihood is $\exp (-\phi)$. Now, if we set phi [i] to $-\log (L(i))$ where the likelihood term $L(i)$ is the contribution of observed perpendicular distance $y[i]$, then the likelihood distribution is clearly found to be $L(i)$. See the WinBUGS manual for further details. The WinBUGS code is as follows:

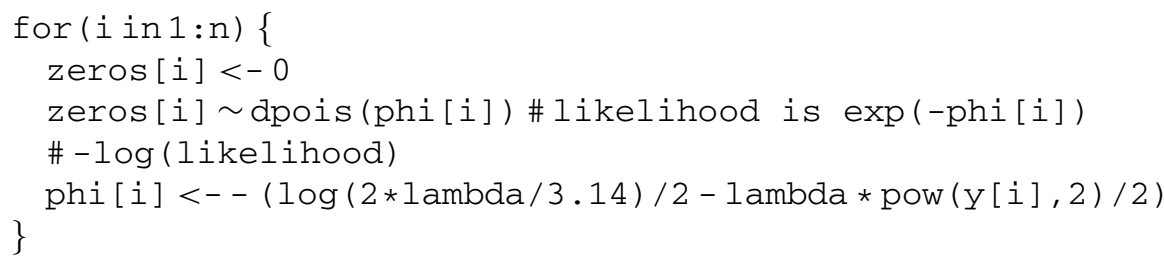

Karunamuni and Quinn (1995) conduct a sensitivity analysis showing that changing values of the prior distribution has little effect on the posterior results. To allow comparisons with Karunamuni and Quinn's results, we use $a=b=0.1$ in our analyses. For parameter $\lambda$, we therefore specify a gamma distribution with both parameters set equal to 0.1 :

lambda $\sim \operatorname{dgamma~}(0.1,0.1)$

Finally, we calculate an estimate of and the density $D$ (Eqs. (8) and (10)):

f $0<-\operatorname{sqrt}(2 * 1$ ambda $/ 3.14)$

$\mathrm{D}<-(\mathrm{n} * \mathrm{f} 0) /(2 * \mathrm{~L})$

The results are given in Table 5 and show close agreement with the Bayesian analysis of Karunamuni and Quinn (1995).

Table 5 Results for the Stakes line transect data analysis

\begin{tabular}{lllll}
\hline & $f(0)$ & $\begin{array}{l}\text { Standard } \\
\text { deviation }\end{array}$ & $D$ & $\begin{array}{l}\text { Standard } \\
\text { deviation }\end{array}$ \\
\hline True & 0.110 & & 0.00375 & \\
Maximum likelihood Estimator & 0.098 & & 0.00332 & \\
Karunamuni and Quinn (1995) & 0.097 & 0.008 & $0.00325^{*}$ & \\
This study & 0.097 & 0.009 & $0.00330^{* *}$ & 0.003301 \\
\hline
\end{tabular}

${ }^{*}$ Relative squared error loss, ${ }^{* *}$ absolute squared error loss. 


\section{State-Space Models of Count Data: Assessing Density Dependence}

In this section, we describe the use of WinBUGS to fit population models of density dependence that simultaneously account for both process and observation error. The example data we use are annual estimates of the population size of North American duck species on their breeding grounds from 1955 to 2002, derived from the Waterfowl Breeding Population and Habitat Survey (WBPHS, US Fish and Wildlife Service 2003).

Assessing the importance of population size or density in regulating population growth rate is fundamental to population biology, ecology and conservation. However, devising robust tests for this so-called "density dependence" has been controversial (e.g. Lebreton this volume). One problem has been that available data on population sizes or densities are almost always estimates, with some level of observation error, and ignoring this observation error can lead to biased tests (e.g. Shenk et al. 1998).

A potential solution is to use a state-space modeling framework, where one can explicitly specify models for both the underlying population dynamics that change population size over time and the observation process that links true population size to the estimates. Such models describing density dependence were constructed by Jamieson (2004) and Jamieson and Brooks (2004). Here we take as an example their "logistic" model for the population dynamics ("state process model"), which can be written as follows:

$$
n_{t}=n_{t-1} \exp \left(\beta_{0}+\sum_{j=1}^{k} \beta_{j} n_{t-j}+\sigma_{p} z_{p, t}\right)
$$

where $n_{t}$ is the population size at time $\mathrm{t}(t=1 \ldots, T), \beta_{0}$ determines the expected rate of population growth when the population size is zero, $\beta_{j}$ determines the rate at which growth is changed depending on population size in time period $t-j, z_{p, t}$ is a Gaussian $\mathrm{N}(0,1)$ random variable that represents un-modeled variation in population growth between time periods ("process error") and $\sigma_{p}$ determines the size of these random fluctuations. This is coupled with an "observation process model", which can be written

$$
y_{t}=n_{t}+s_{o, t} z_{o, t}
$$

where $y_{t}$ is the estimated population size at time $\mathrm{t}, z_{o, t}$ is a Gaussian $\mathrm{N}(0,1)$ random variable that represents measurement error and $s_{o, t}$, which is assumed known (it is provided as part of the WBPHS data, for example), determines the size of the measurement errors.

The state-space model defined by Eqs. (11) and (12) is non-linear and nonnormal (because of Eq. (11)), and therefore is difficult to fit using standard frequentist methods, such as the Kalman filter (although see de Valpine 2002, 2003; 
de Valpine and Hastings 2002; Besbeas et al. 2005; Besbeas et al. this volume). Jamieson (2004) and Jamieson and Brooks (2004) describe how the model can be formulated in a Bayesian context, and how the parameters may be estimated, for fixed $k$, using MCMC. Further, they show how a recent extension of the MCMC algorithm - Reversible Jump MCMC (RJMCMC; Green 1995) - can be used to compute the posterior probability for each of a set of possible values of $k$, and thereby estimate the probability of the presence of density dependence (i.e., the probability that $k>0$ ) in a population (although we note that autocorrelated process error can affect such assessments - see Lebreton this volume). For the use of RJMCMC in population ecology, see for example, King and Brooks (2002a, b, 2003, 2008) and King et al. (2006). RJMCMC can also be used to produce model-averaged predictions of future population size. Jamieson and Brooks (2004) apply these methods using custom-written MCMC and RJMCMC samplers, implemented in the computer language $\mathrm{C}$, to data for 10 species of duck from the WBPHS. Three species (Northern Pintail Anas acuta, Redhead Aythya americana and Canvasback Aythya valisineria) appear to show some form of density dependence.

Similar models were fitted to Canvasback and Mallard data from the WBPHS (as well as simulated data) by Viljugrein et al. (2005) using WinBUGS, although code was not included with that paper. An additional covariate, number of breeding ponds, was included and model discrimination was via DIC. In that paper, both species were found to show density dependence.

Our aim is to demonstrate how these models may be fitted using WinBUGS, to investigate the use of the beta version of the RJMCMC plug-in for WinBUGS, and to validate the results by comparing them with the independent sampler and $\mathrm{C}$ code written by Jamieson. We present some of this work here; it is described in detail in Parker et al. (in prep.). To save space, we only present results for Canvasback.

\subsection{Logistic Model}

For computational convenience, we re-parameterized the model presented above so that time periods $t=1, \ldots, k$ are the times before data are available and $t=k+1$, $\ldots, k+T$ are times when data were collected. Note that missing data are easily accommodated in this framework. We also turned Eq. (11) into an additive model by log-transforming:

$$
p_{t}=p_{t-1}+\beta_{0}+\sum_{j=1}^{k} \beta_{j} \exp \left(p_{n-j}\right)+\sigma_{p} z_{t}
$$

where $p_{t}=\log \left(n_{t}\right)$.

Bayesian methods require specification of prior distributions on all unknown quantities; for the purposes of comparison we used exactly the same distributions as used in Chapter 2 of Jamieson (2004; note these are slightly different from 
those of Jamieson and Brooks 2004): $\beta_{j} \sim N(0,100)$ for $j=0, \ldots, k, \sigma_{p}^{2}=$ $\Gamma^{-1}(0.001,0.001)$ and $n_{t} \sim N(0.540,0.130)$ for $t=1, \ldots, k$. Note that numbers of ducks are expressed $\times 10^{6}$ and that the distribution is truncated so that $n_{t}>0$ (by setting all sampled values of $n_{t}$ to the maximum of the value drawn from the above normal distribution and 0.00001). Priors are not required on $n_{t}, t=k+1, \ldots, k+T$ due to the Markovian structure of the state process model: priors for these quantities are implicitly specified when priors are set for $n_{t}, t=1, \ldots, k$. (See Jamieson 2004 for an in-depth discussion of this; see also de Valpine 2002 and Maunder et al. this volume).

Our WinBUGS program was based on code originally written by Steve Brooks for a workshop on Bayesian methods (Brooks et al. 2005). The key parts are specification of the observation process equation (Eq. (12)) and system process equation (Eq. (13)). The observation process equation code is:

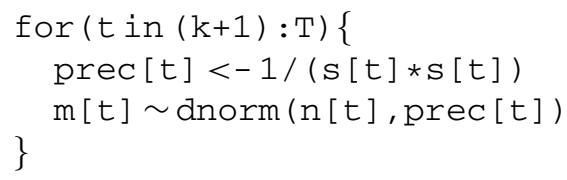

while the system process equation code is:

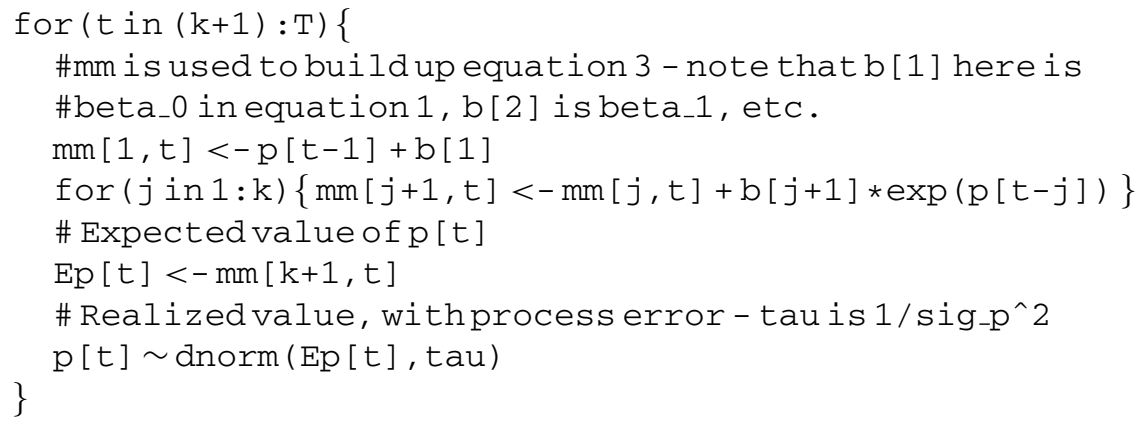

Predictions of future states, for example up to time $T+10$, could easily be obtained by replacing the first line of the above loop with

for $(t$ in $(k+1):(T+10))\{$

Summaries of the posterior parameter estimates for Canvasback for $k=1,2$, and 5 and runs with burn-in of 50,000 and then 1,000,000 samples are given in Table 6, as are results from the same model reproduced from Jamieson (2004, Table 2.5).

The results are very similar, with differences within the bounds of Monte-Carlo variation. Convergence and mixing were relatively slow; diagnostics are reported in Parker et al. (in prep.).

A naïve way to look for evidence of density dependence is to examine posterior credibility intervals $(\mathrm{CI})$ on the $\beta$ parameters. For example, in the first-order time lag model $(k=1)$, the $95 \%$ posterior CI does not contain 0 throughout, providing support for the notion of first order density dependence in this species. 


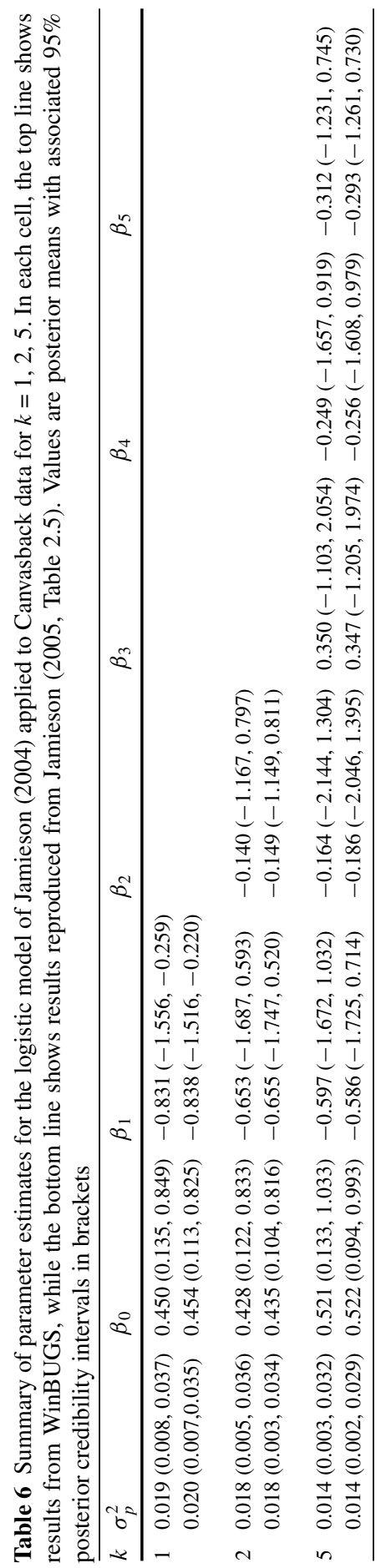




\subsection{Model Comparison}

The above program was extended to allow selection among models using RJMCMC. This algorithm searches over the different models, given the observed data, so that the number of possible models is no longer restrictive. We consider an extension to the standard Bayes Theorem, where we simply consider the model itself to be a (discrete) parameter. The standard formula still applies, but now the posterior distribution is defined over both the parameter and model space. Integrating over the parameters we are able to calculate the marginalized posterior probability for each model. However, this integration is analytically intractable and so we resort to an MCMC-type approach. The standard MCMC algorithm cannot be used in the presence of model uncertainty, and RJMCMC is therefore used to explore simultaneously the parameter and model space within a single Markov chain. We used the Jump extension to WinBUGS (Lunn et al. 2006) to implement RJMCMC. This extension allows the sampler to move between models that include all possible combinations of a set of potential covariates - in our case $\beta_{1}$ to $\beta_{V}$ where $V$ is the maximum time lag allowable (set to 5 in our code). $k$ indexes the number of $\beta$ parameters (excluding $\beta_{0}$, which is in all models) in the model for a particular draw from the chain (i.e., the dimension of the model). In the code, an indicator variable id, indicates which particular model is in a particular draw - for example if id was 10101, that would indicate that the parameters $\beta_{1}, \beta_{3}$, and $\beta_{5}$ were in the model for that draw (and therefore that $k=3$ ).

In the Jump protocol one specifies a prior on the models by specifying a prior distribution on $k$. The following gives a prior probability of 0.5 that any $\beta_{j}(0>j \geq$ $V$ ) is in the model (Lunn 2006, p. 3):

$\mathrm{k} \sim \operatorname{dbin}(0.5, \mathrm{~V})$

We then specify a design matrix (see Lunn 2006, Eq. (1) with the number of rows equal to the number of time periods and $\mathrm{V}$ columns. The elements of each row correspond to the sum in Eq. (13). In the following code, $\mathrm{C}$ is the first time period about which we make posterior inferences in states - i.e., $C=\mathrm{V}+1$.

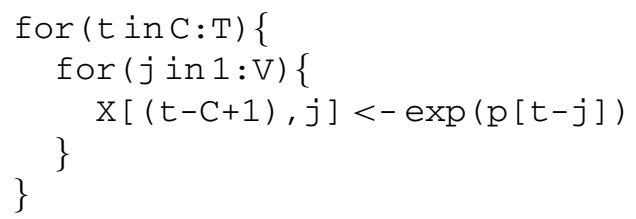

To set up the reversible jump, we use the two Jump-specific commands jump. Iin.pred and jump.model.id, as follows:

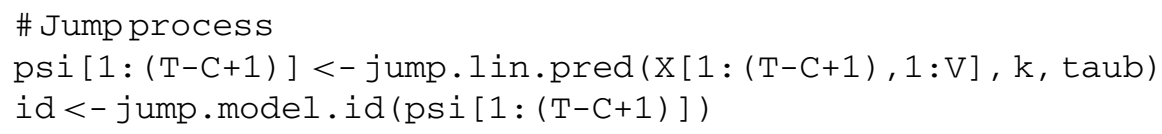

where psi is a vector representing the current values of the linear predictor (Lunn 2006, Eq. (1), and taub is the prior precision on the $\beta$ parameters (in our case 
$1 / 100$; note that the prior on all $\beta$ parameters is assumed to be multivariate normal, with mean 0 and the specified precision - this distribution is fixed by the software).

We note that the priors specified on the parameters can influence the corresponding posterior model probabilities. In other words the posterior model probabilities are often sensitive to the prior parameter specification. Thus we recommend that a prior sensitivity analysis should always be performed, and care taken when specifying the priors for the parameters, to represent sensible prior beliefs.

Lastly, we specify the system process equation in terms of the psi variable:

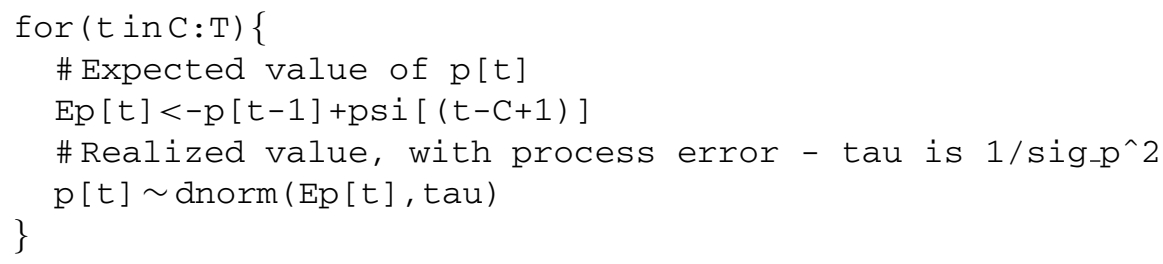

Posterior model probabilities can be calculated from the proportion of time the chain visited each model of interest. This information can be obtained from the Jump menu that is added to the WinBUGS interface when the Jump extension is installed, and reports the proportion of time spent in each value of the id variable. Note, however, some of the models included in the chain are not of interest - we are only interested in models that for any given $k$ contain parameters $\beta_{1}, \ldots, \beta_{k}$ : for example with $k=2$ we are only interested in id 11000, and not 10100, 01100, etc. We therefore select out from the list of id's only those we are interested in, and re-normalize so that the proportion of times in these models of interest sum to 1 . These proportions are then estimates of posterior model probability.

Model-averaged estimates of other unknown quantities, such as the $n_{t} \mathrm{~s}$, can also be produced by WinBUGS, but just as with the ids above, these contain both models we are interested in and those we are not. It is necessary to save the value for the variable of interest generated in each sample (the CODA button in the sample monitor tool will do this), as well as the corresponding id values, and then select out only those samples that were generated under id values corresponding to models of interest.

Posterior model probabilities for Canvasback for runs with burn-in of 50,000 and then 1,000,000 samples are given in Table 7, as are results from the equivalent model from a run of the Jamieson C code using burn-in of 20,000 and 100,000 samples

Table 7 Posterior model probabilities for $k=0, \ldots, 5$ for the logistic model of Jamieson (2004) applied to Canvasback data

\begin{tabular}{lll}
\hline$k$ & WinBUGS & Jamieson C code \\
\hline 0 & 0.279 & 0.265 \\
1 & 0.685 & 0.697 \\
2 & 0.034 & 0.036 \\
3 & 0.002 & 0.002 \\
4 & 0.000 & 0.001 \\
5 & 0.000 & 0.000 \\
\hline
\end{tabular}


(a run of the Jamieson code was required because posterior model probabilities were not given for these priors in Jamieson 2004).

While the results are similar, they are not identical. This is likely to be caused by a small difference between the implementations: in the algorithm of Jamieson, the acceptance probabilities for between-model moves do not depend on the priors for the $\beta$ parameters (Jamieson 2005, Section 3.1.1), while in the WinBUGS algorithm it is not possible to achieve such tuning, and in the default algorithm the priors on the $\beta$ parameters do affect acceptance rates. Despite these minor differences, the overall conclusions are the same: the best supported model (posterior model probability $0.6-0.7$ ) is the one with first-order density dependence.

\section{Discussion}

In this paper, we have seen how Bayesian theory can be applied to stochastic models for population ecology using MCMC algorithms as implemented in program WinBUGS.

In a mark-recapture data modeling context, WinBUGS can handle many complex models, without additional effort once the likelihood has been written down. This includes (i) random effects that allow unexplained residual variance to be coped with when dealing with covariates, automatic calculation of the amount of smoothing when splines are to be used but also temporal autocorrelation to be incorporated (Johnson and Hoeting 2003), (ii) missing data in the covariate values to be handled and (iii) variable selection. Note that those advantages may also be applied in distance sampling models in order to incorporate covariates in the modeling of the detection function (Marques and Buckland 2003; Marques et al. 2007). Random effects can also be used to address spatial variation in both families of models, allowing the survival and the detection function to depend on spatial coordinates (e.g. longitude and latitude) using splines in two dimensions (Gimenez and Barbraud this volume) or a combination of various random effects (Grosbois et al. in revision) or alternatively, using the geostatistical tools as available through the GeoBUGS adds-on of WinBUGS and the possibility of interfacing WinBUGS with Geographic Information System (GIS) software (WinBUGS manual; see Wyatt 2003 for an application in fisheries).

In our experience, using $\mathrm{R}$ or MATLAB to call WinBUGS makes its use much easier for pre- and post-processing data. Note also that an open-source version of the WinBUGS code has recently been published as OpenBUGS. Among other advances, it can be made to perform block updates (i.e., update multiple unknown quantities simultaneously), which might be of interest for experienced programmers. OpenBUGS also runs under Linux.

Our introduction may make WinBUGS appear like a panacea. However, like all computer programs, WinBUGS is not always the perfect tool for Bayesian methods in population ecology, and developments are taking place to improve it. However, as can be appreciated from the three case-studies, it is capable of producing informative results for sophisticated models. In using WinBUGS, one should be aware 
of the following potential problems. First, one should be aware that experience is needed to be able to debug WinBUGS programs. Also, the computational burden may be discouraging, and it is sometimes preferable to resort to Fortran or C++ to implement efficient MCMC algorithms for specific problems. Finally, although user-specific functions can be programmed (see the WinBUGS manual), there are no tools for matrix calculus so that, e.g., multistate mark-recapture models are difficult to implement (see however Durban et al. 2005 for closed populations). Interestingly, a state-space modeling approach for data on marked animals proposed by Gimenez et al. (2007) might be a solution to this problem (see also Royle [in press] for a similar state-space formulation allowing modeling individual effects). More generally, in line with Buckland et al. (2004b; see also Newman et al. 2006; Buckland et al. 2007), we believe that state-space modeling can provide a convenient and flexible framework for specifying many stochastic models for the dynamics of wild animal populations. In doing so, WinBUGS may provide an efficient and flexible tool to fit such models, possibly nonlinear and non Gaussian - as has been realized for several years in fisheries (Meyer and Millar 1999; Millar and Meyer 2000; Rivot and Prévost 2002; Lewy and Nielsen 2003; Rivot et al. 2004). We note that other fitting algorithms, such as variations on the Kalman filter, Monte-Carlo particle filter, Laplace approximation, importance sampling may also be applicable (see Buckland et al. 2007 for a review). These ideas open the area to numerous applications including the integration of several sources of information (recovery and recapture data, see Catchpole et al. 1998; count data and demographic data, see Besbeas et al. 2002, 2005, 2008; Brooks et al. 2004; Maunder 2004; Schaub et al. 2007).

We end by providing a non-comprehensive list of applications of Bayesian methods in population ecology. An important advantage of the Bayesian framework is the possibility to incorporate prior information in the analysis. McCarthy and Masters (2005a) show how to use prior information on body mass to improve survival estimates using the CJS models, while Pearce et al. (2001); Yamada et al. (2003); Kuhnert et al. (2005) and Martin et al. (2005a) show how to integrate expert knowledge. Several authors have dealt with important issues regarding the specification of vague priors (Lambert et al. 2005; van Dongen 2006; Gelman 2006), assessment of the sensitivity of the posterior distribution to the specified prior distribution (Millar 2004; Millar and Stewart 2005), parameter identifiability in a Bayesian context (Gimenez et al. this volume) and goodness-of-fit tests (Brooks et al. 2000; Barry et al. 2003; Michielsens and McAllister 2004). Meta-analyses have been successfully carried out to estimate demographic parameters (Tufto et al. 2000) and assess animal movement (Jonsen et al. 2003). Further applications of WinBUGS to analyze animal movement data can be found in Morales et al. (2004) and Jonsen et al. (2005). WinBUGS can be used to address issues associated with binomial and Poisson data such as spatial autocorrelation (Thogmartin et al. 2004; Wintle and Bardos 2006), imperfect detection (Royle and Dorazio 2006), heterogeneity in the detection process (Durban and Elston 2005), excess of zeros (Martin et al. 2005b; Ghosh et al. 2006), observer effects (Thogmartin et al. 2004), detecting trends (Link and Sauer 2002) and missing data (Lens et al. 2002). WinBUGS has 
allowed a better understanding of the impact in assessing complex effects of densitydependence and predicting the impact of climate change and human exploitation in population dynamics (Bjornstad et al. 1999; Saether et al. 2000; Stenseth et al. 2003; Conroy et al. 2005). Regarding model selection, alternatives to DIC and RJMCMC using WinBUGS are given by Ntzoufras (2002; Gibbs variable selection), Link and Barker (2006; Bayesian information criterion; see also Link and Barker this volume) and Ghosh and Norris (2005; minimum posterior predictive loss). Finally, Link and Barker (2005) considered association among demographic parameters (e.g. recruitment and survival) in analysis of open population mark-recapture data (see also Cam et al. 2002 and Wintrebert et al. 2005 when detectability is equal to one).

In conclusion, we hope this paper will encourage ecologists to explore the potential of the flexible and useful WinBUGS software, and the methods underlying it, for carrying out future applications.

\section{References}

Bairlein F (1991) Population studies of white storks ciconia ciconia in Europe, with reference to the western population. In: Perrins C, Lebreton J-D, Hirons G (eds), Bird Population Studies: Relevance to Conservation and Management, pages 207-229. Oxford University Press. Oxford.

Barbraud C, Barbraud JC, Barbraud M (1999) Population dynamics of the White Stork Ciconia ciconia in western France. Ibis 141:469-479.

Barry SC, Brooks SP, Catchpole EA, Morgan BJT (2003) The analysis of ring-recovery data using random effects. Biometrics 58:54-65.

Besbeas P, Freeman SN, Morgan BJT, Catchpole EA (2002) Integrating mark-recapture-recovery and census data to estimate animal abundance and demographic parameters. Biometrics 58:540-547.

Besbeas P, Freeman SN, Morgan BJT (2005) The potential of integrated population modelling. Australian New Zealand Journal of Statistics 47:35-48.

Biller C (2000) Adaptive Bayesian Regression Splines in semiparametric generalized linear models. Journal of Computational and Graphical Statistics 9:122-140.

Bjornstad ON, Fromentin JM, Stenseth NC, Gjosaeter J (1999) Cycles and trends in cod populations. Proceedings of the National Academy of Sciences of the USA 96:5066-5071.

Bonner SJ, Schwarz CJ (2006) An extension of the Cormack-Jolly-Seber model for continuous covariates with applications to Microtus pennsylvanicus. Biometrics 62:142-149.

Brooks SP, Catchpole EA, Morgan BJT (2000) Bayesian animal survival estimation. Statistical Science 15:357-376.

Brooks SP, Catchpole EA, Morgan BJT, Harris MP (2002) Bayesian methods for analysing ringing data. Journal of Applied Statistics 29:187-206.

Brooks SP, King R, Morgan BJT (2004) A Bayesian approach to combining animal abundance and demographic data. Animal Biodiversity and Conservation 27:515-529.

Brooks SP, Gimenez O, King R (2005) Bayesian methods for population ecology - workshop codes. National Centre for Statistical Ecology Software Library. [http://www. ncse.org.uk/software.html].

Buckland ST, Anderson DR, Burnham KP, Laake JL, Borchers DL, Thomas L (2001) Introduction to Distance Sampling. Oxford University Press, Oxford.

Buckland ST, Anderson DR, Burnham KP, Laake JL, Borchers DL, Thomas L Eds. (2004a) Advanced Distance Sampling. Oxford University Press, Oxford. 
Buckland ST, Newman KB, Thomas L, Koesters NB (2004b) State-space models for the dynamics of wild animal populations. Ecological Modelling 171:157-175.

Buckland ST, Newman KB, Fernández C, Thomas L, Harwood J (2007) Embedding population dynamics models in inference. Statistical Science 22:44-58.

Burnham KP, White GC (2002) Evaluation of some random effects methodology applicable to bird ringing data. Journal of Applied Statistics 29:245-264.

Cam E, Link WA, Cooch EG, Monnat JY, Danchin E (2002) Individual covariation between lifehistory traits: seeing the trees despite the forest. The American Naturalist 159:96-105.

Catchpole EA, Freeman SN, Morgan BJT, Harris MJ (1998) Integrated reovery/recapture data analysis. Biometrics 54:33-46.

Celeux G, Forbesy F, Robert CP, Titterington DM (2006) Deviance information criteria for missing data models. Bayesian Analysis 1:651-674.

Chavez-Demoulin V (1999) Bayesian inference for small-sample capture-recapture data. Biometrics 55:727-731.

Choquet R, Reboulet A-M, Pradel R, Gimenez O, Lebreton J-D (2005) M-SURGE: new software specifically designed for multistate capture-recapture models. Animal Biodiversity and Conservation, 27: 207-215. Available from http://www.cefe.cnrs.fr/biom/logiciels.htm/

Clark JS (2005) Why environmental scientists are becoming Bayesians. Ecology Letters 8:2-14.

Clobert J, Lebreton J-D (1985) Dépendance de facteurs de milieu dans les estimations de taux de survie par capture-recapture. Biometrics 412:1031-1037.

Congdon P (2003) Applied Bayesian Modelling, John Wiley, New York.

Congdon P (2006) Bayesian Statistical Modelling, 2 nd edition. John Wiley, New York.

Conroy MJ, Fonnesbeck CJ, Zimpfer NL (2005) Modeling regional waterfowl harvest rates using Markov Chain Monte Carlo. Journal of Wildlife Management 69:77-90.

Cormack RM (1964) Estimates of survival from sighting of marked animals. Biometrika 51:429-438.

Crainiceanu CM, Ruppert D, Wand MP (2005) Bayesian analysis for penalized spline regression using WinBUGS. Journal of Statistical Software 14:1-24.

de Valpine P (2002) Review of methods for fitting time-series models with process and observation error, and likelihood calculations for nonlinear, non-Gaussian state-space models. Bulletin of Marine Science 70:455-471.

de Valpine P (2002) Review of methods for fitting time-series models with process and observation error and likelihood calculations for nonlinear, non-Gaussian state-space models. Bulletin of Marine Science 70:455-471.

de Valpine P (2003) Better inferences from population-dynamics experiments using Monte Carlo state-space likelihood methods. Ecology 84:3064-3077.

de Valpine P, Hastings A (2002) Fitting population models incorporating process noise and observation error. Ecological Monographs 72:57-76.

Durban JW, Elston DA (2005) Mark-recapture with occasion and individual effects: abundance estimation through Bayesian model selection in a fixed dimensional parameter space. Journal of Agricultural, Biological, and Environmental Statistics 10:291-305.

Durban JW, Elston DA, Ellifrit DK, Dickson E, Hammond PS, Thompson P (2005) Multisite mark-recapture for cetaceans: population estimates with Bayesian model averaging. Marine Mammal Science 21:80-92.

Ellison AM (2004) Bayesian inference in ecology. Ecology Letters 7:509-520.

Fournier D (2001) An introduction to AD MODEL BUILDER Version 6.0.2 for use in nonlinear modeling and statistics. Available from http://otter-rsch.com/admodel.htm.

Gelman A (2006) Prior distributions for variance parameters in hierarchical models (Comment on Article by Browne and Draper). Bayesian Analysis 1:515-534.

Ghosh SK, Norris JL (2005) Bayesian capture-recapture analysis and model selection allowing for heterogeneity and behavioral effects. Journal of Agricultural, Biological, and Environmental Statistics 10:35-49.

Ghosh SK, Mukhopadhyay P, Lu J-C (2006) Bayesian analysis of zero-inflated regression models. Journal of Statistical Planning and Inference 136:1360-1375. 
Gilks W, Richardson S, Spiegelhalter D (1996) Markov chain Monte Carlo in practice. Chapman \& Hall, London.

Gimenez O, Crainiceanu C, Barbraud C, Jenouvrier S, Morgan BJT (2006a) Semiparametric regression in capture-recapture modelling. Biometrics 62:691-698.

Gimenez O, Covas R, Brown CR, Anderson MD, Bomberger Brown M, Lenormand T (2006b) Nonparametric estimation of natural selection on a quantitative trait using mark-recapture data. Evolution 60:460-466.

Gimenez O, Rossi V, Choquet R, Dehais C, Doris B, Varella H, Vila J-P, Pradel R (2007) Statespace modeling of data on marked individuals. Ecological Modelling, 206:431-438.

Gimenez O, Barbraud C (2008) The efficient semiparametric regression modelling of capturerecapture data: assessing the impact of climate on survival of two Antarctic seabird species. In: Thomson DL, Cooch EG, Conroy MJ (eds.) Modeling Demographic Processes in Marked Populations. Environmental and Ecological Statistics, Springer, New York.

Gimenez BJT, Morgan O, Brooks SP (2008) Weak identifiability in models for mark-recapturerecovery data. In: Thomson DL, Cooch EG, Conroy MJ (eds.) Modeling Demographic Processes in Marked Populations. Environmental and Ecological Statistics, Springer, New York.

Gould WR, Nichols JD (1998) Estimation of temporal variability of survival in animal populations. Ecology 79:2531-2538.

Green PJ (1995) Reversible jump MCMC computation and Bayesian model determination. Biometrika 82:711-732.

Grosbois V, Gimenez O, Gaillard J-M, Pradel R, Barbraud C, Clobert J, Møller AP, Weimerskirch $\mathrm{H}$ (in revision) Assessing the impact of climate variation on survival in vertebrate populations. Biological Reviews.

Grosbois V, Harris MP, Anker-Nilssen T, McCleery RH, Shaw DN, Morgan BJT, Gimenez O (in revision) Spatial modelling of survival using capture-recapture data. Ecology.

Harley SJ, Myers RA, Field CA (2004) Hierarchical models improve abundance estimates: spawning biomass of hoki in Cook Strait, New Zealand. Ecological Applications 14: 1479-1494.

Ihaka SP, Gentleman EA (1996) R: a language for data analysis and graphics. Journal of Computational and Graphical Statistics 5:299-314.

Jamieson LE (2004) Bayesian model discrimination with application to population ecology and epidemiology. PhD Thesis, University of Cambridge.

Jamieson LE, Brooks SP (2004) Density dependence in North American ducks. Animal Biodiversity and Conservation 27:113-128.

Johnson DS, Hoeting JA (2003) Autoregressive models for capture-recapture data: a Bayesian approach. Biometrics 59:341-350.

Jolly GM (1965) Explicit estimates from capture-recapture data with both death and immigrationstochastic model. Biometrika 52:225-247.

Jonsen ID, Myers RA, Flemming JM (2003) Meta-analysis of animal movement using state-space models. Ecology 84:3055-3063.

Jonsen ID, Flemming JM, Myers RA (2005) Robust state-space modeling of animal movement data. Ecology 86:2874-2880.

Kanyamibwa S, Schierer A, Pradel R, Lebreton JD (1990) Changes in adult survival rates in a western European population of the White Stork Ciconia ciconia. Ibis 132:27-35.

Karunamuni RJ, Quinn II TJ (1995) Bayesian estimation of animal abundance for the line transects sampling. Biometrics 51:1325-1337.

Kass RE, Carlin BP, Gelman A, Neal RM (1998) Markov chain Monte Carlo in practice: a roundtable discussion. American Statistician 52:93-100.

King R, Brooks SP (2002a) Bayesian model discrimination for multiple strata capture-recapture data. Biometrika 89:785-806.

King R, Brooks SP (2002b) Model selection for integrated recovery/recapture data. Biometrics 58:841-851. 
King R, Brooks SP (2003) Survival and spatial fidelity of mouflons: the effect of location, age and sex. Journal of Agricultural, Biological and Environmental Statistics 8:486-513.

King R, Brooks SP, Morgan BJT, Coulson T (2006) Bayesian analysis of factors affecting soay sheep. Biometrics 62:211-220.

King R, Brooks SP (2008) On the Bayesian estimation of a closed population size in the presence of heterogeneity and model uncertainty. Biometrics, In press.

Kuhnert PM, Martin TG, Mengersen K, Possingham HP (2005) Assessing the impacts of grazing levels on bird density in woodland habitat: a Bayesian approach using expert opinion. Environmetrics 16:717-747.

Lambert PC, Sutton AJ, Burton PR, Abrams KR, Jones DR (2005) How vague is vague? A simulation study of the impact of the use of vague prior distributions in MCMC using WinBUGS. Statistics in Medicine 24:2401-2428.

Lebreton J-D, Burnham KP, Clobert J, Anderson DR (1992) Modeling survival and testing biological hypothesis using marked animals: a unified approach with case studies. Ecological Monographs 62:67-118.

Lebreton J-D (2008) Assessing density-dependence: where are we left? In: Thomson DL, Cooch EG, Conroy MJ (eds.) Modeling Demographic Processes in Marked Populations. Environmental and Ecological Statistics, Springer, New York.

Lens L, van Dongen S, Norris K, Githiru M, Matthysen E (2002) Avian persistence in fragmented rainforest. Science 298:1236-1238.

Lewy P, Nielsen A (2003) Modeling stochastic fish stock dynamics using Markov Chain Monte Carlo. ICES Journal of Marine Science 60:743-752.

Link WA, Cam E, Nichols JD, Cooch EG (2002) Of bugs and birds: Markov Chain Monte Carlo for hierarchical modelling in wildlife research. Journal of Wildlife Management 66:277-291.

Link WA, Sauer JR (2002) A hierarchical model of population change with application to cerulean warblers. Ecology 83:2832-2840.

Link WA, Barker RJ (2005) Modeling association among demographic parameters in analysis of open population capture-recapture data. Biometrics 61:46-54.

Link WA, Barker RJ (2006) Model weights and the foundations of multimodel inference. Ecology 87:2626-2635.

Lunn DJ (2006) WinBUGS 'jump' interface: beta-release user manual. Technical report dated February 14th 2006, School of Medicine, Imperial College London, UK.

Lunn DJ, Whittaker JC, Best N (2006) A Bayesian toolkit for genetic association studies. Genetic Epidemiology 30:231-247.

Marques FFC, Buckland ST (2003) Incorporating covariates into standard line transect analyses. Biometrics 59:924-935.

Marques TA, Thomas L, Fancy SG, Buckland ST (2007) Improving estimates of bird density using multiple covariate distance sampling. The Auk 124:1229-1243.

Martin TG, Kuhnert PM, Mengessen K, Possingham HP (2005a) The power of expert opinion in ecological models using Bayesian methods: Impact of grazing on birds. Ecological Applications 15:266-280.

Martin TG, Wintle BA, Rhodes JR, Kuhnert PM, Field SA, Low-Choy SJ, Tyre AJ, Possingham HP (2005b) Zero tolerance ecology: improving ecological inference by modelling the source of zero observations. Ecology Letters 8:1235-1246.

Maunder MN (2004) Population viability analysis based on combining Bayesian, integrated, and hierarchical analyses. Acta Oecologica 26:85-94.

Maunder MN, Schnute JT, Ianelli JN (in press) Computers in fisheries population dynamics. In: Megrey B, Moksness E (eds) Computers in Fisheries Research. Kluwer Academic Publishers, Dordrecht.

Maunder MN, Skaug HJ, Hoyle SD (2008) Comparison of estimators for mark-recapture models: random effects, hierarchical Bayes, and AD Model Builder. In: Thomson DL, Cooch EG, Conroy MJ (eds.) Modeling Demographic Processes in Marked Populations. Environmental and Ecological Statistics, Springer, New York. 
McCarthy MA, Masters P (2005) Profiting from prior information in Bayesian analyses of ecological data. Journal of Applied Ecology 42:1012-1019.

McCarthy MA (2007) Bayesian Methods for Ecology. Cambridge University Press, Cambridge.

Meyer R, Millar RB (1999) Bugs in bayesian stock assessments. Canadian Journal of Fisheries Aquatic Sciences 56:1078-1087.

Michielsens CGJ, McAllister MK (2004) A Bayesian hierarchical analysis of stock-recruit data: quantifying structural and parameter uncertainties. Canadian Journal of Fisheries Aquatic Sciences 61:1032-1047.

Millar RB, Meyer R (2000) Bayesian state-space modeling of age-structured data: fitting a model is just the beginning. Canadian Journal of Fisheries Aquatic Sciences 57:43-50.

Millar RB (2004) Sensitivity of Bayes estimators to hyper-parameters with an application to maximum yiel from fisheries. Biometrics 60:536-542.

Millar RB, Stewart WS (2005) Automatic calculation of the sensitivity of Bayesian fisheries models to informative priors. Canadian Journal of Fisheries and Aquatic Sciences 62: $1028-1036$.

Morales JM, Haydon DT, Frair J, Holsinger KE, Fryxell JM (2004) Extracting more out of relocation data: building movement models as mixtures of random walks. Ecology 85:2436-2445.

Mysterud A, Stenseth NC, Yoccoz NG, Langvatn R, Steinheim G (2001) Nonlinear effects of large-scale climatic variability on wild and domestic herbivores. Nature 410: 1096-1099.

Newman KB, Buckland ST, Lindley ST, Thomas L, Fernández C (2006) Hidden process models for animal population dynamics. Ecological Applications 16:74-86.

North PM, Morgan BJT (1979) Modeling heron survival using weather data. Biometrics 35: $667-681$.

Ntzoufras I (2002) Gibbs variable selection using BUGS. Journal of Statistical Software, 7(7).

Pearce JL, Cherry K, Drielsma M, Ferrier S, Whish G (2001) Incorporating expert opinion and fine-scale vegetation mapping into statistical models of faunal distribution. Journal of Applied Ecology 38:412-424.

Plummer M, Best NG, Cowles K, Vines K (2004) CODA: output analysis and diagnostics for MCMC. R package version 0.9-1, URL http://www-fis.iarc.fr/coda/.

Pollock KH, Hines JE, Nichols JD (1984) The use of auxiliary variables in capture-recapture and removal experiments. Biometrics 40:329-340.

Pollock KH (2002) The use of auxiliary variables in capture-recapture modelling: an overview. Journal of Applied Statistics 29:85-102.

R Development Core Team (2007) R: a language and environment for statistical computing. R Foundation for Statistical Computing, Vienna, Austria. ISBN 3-900051-07-0, URL http://www.R-project.org.

Rivot E, Prévost E (2002) Hierarchical Bayesian analysis of capture-mark-recapture data. Canadian Journal of Fisheries Aquatic Sciences 59:1768-1784.

Rivot E, Prévost E, Parent E, Baglinière JL (2004) A Bayesian state-space modelling framework for fitting a salmon stage-structured population dynamic model to multiple time series of field data. Ecological Modelling 179:463-485.

Royle JA, Link WA (2002) Random effects and shrinkage estimation in capture-recapture models. Journal of Applied Statistics 29:329-351.

Royle JA, Dorazio RM (2006) Hierarchical models of animal abundance and occurrence. Journal of Agricultural, Biological, and Environmental Statistics 11:249-263.

Royle JA (2008) Modeling individual effects in the Cormack-Jolly-Seber model: a state-space formulation. Biometrics, In press.

Ruppert D, Wand MP, and Carroll R (2003) Semiparametric Regression. Cambridge University Press, Cambridge.

Saether BE, Tufto J, Engen S, Jerstad K, Rostad OW, Skatan JE (2000) Population dynamical consequences of climate change for a small temperate songbird. Science 287:854-856.

Schaub M, Kania W, Koppen U (2005) Variation of primary production during winter induces synchrony in survival rates in migratory white storks Ciconia ciconia. Journal of Animal Ecology 74:656-666. 
Schaub M, Gimenez O, Sierro S, Arlettaz R (2007) Assessing population dynamics from limited data with integrated modeling: life history of the endangered greater horseshoe bat. Conservation Biology 21:945-955.

Seber GAF, (1965) A note on multiple-recapture census. Biometrika 52:249-259.

Shenk TM, White GC, Burnham KP (1998) Sampling-variance effects on detecting density dependence from temporal trends in natural populations. Ecological Monographs 68:445-463.

Skaug HJ, Fournier DA (2006) Automatic approximation of the marginal likelihood in nonGaussian hierarchical models. Computational Statistics and Data Analysis 51:699-709.

Smith BJ (2004) BOA: Bayesian Output Analysis Program (BOA) for MCMC. R package version 1.1.2-1, URL http://www.public-health.uiowa.edu/boa.

Spiegelhalter DJ, Best NG, Carlin BR, van der Linde A (2002) Bayesian measures of model complexity and fit. Journal of the Royal Statistical Society Series B-Statistical Methodology 64:583-616.

Spiegelhalter D, Thomas A, Best N, Lunn D (2003) WinBUGS user manual. version 1.4 (http://www.mrc-bsu.cam.ac.uk/bugs.). Technical report, Medical Research Council Biostatistics Unit. Cambridge.

Stenseth NC, Viljugrein H, Saitoh T, Hansen TF, Kittilsen MO, Bølviken E, Glöckner F (2003) Seasonality, density dependence, and population cycles in Hokkaido voles. Proceedings of the National Academy of Sciences of the USA 100:11478-11483.

Sturtz S, Ligges U, Gelman A (2005) R2WinBUGS: a package for running WinBUGS from R. Journal of Statistical Software 12:1-16.

Thogmartin WE, Sauer JR, Knutson MG (2004) A hierarchical spatial model of avian abundance with application to cerulean warblers. Ecological Applications 14:1766-1779

Tufto J, Saether B-E, Engen S, Arcese P, Jerstad K, Rostad OW, Smith JNM (2000) Bayesian metaanalysis of demographic parameters in three small, temperate passerines. Oikos 88:273-281.

US Fish and Wildlife Service (2003) Waterfowl Population Status, 2003, US Department of the Interior, Washington, DC.

van Dongen S (2006) Prior specification in Bayesian statistics: three cautionary tales. Journal of Theoretical Biology 242:90-100.

Viljugrein H, Stenseth NC, Smith GW, Steinbakk GH (2005) Density dependence in North American ducks. Ecology 86:245-254.

White GC, Burnham KP (1999) Program MARK: survival estimation from populations of marked animals. Bird Study 46:120-129. Available from http://www.cnr.colostate. edu/gwhite/software.html.

Williams BK, Nichols JD, Conroy MJ (2002) Analysis and Management of Animal Populations. Academic Press, New York.

Wintle BA, Bardos DC (2006) Modeling species-habitat relationships with spatially autocorrelated observation data. Ecological Applications 16:1945-1958.

Wintrebert C, Zwinderman AH, Cam E, Pradel R, van Houwelingen JC (2005) Joint modeling of breeding and survival of Rissa tridactyla using frailty models. Ecological Modelling 181: $203-213$.

Wyatt RJ (2003) Mapping the abundance of riverine fish populations: integrating hierarchical Bayesian models with a geographic information system (GIS). Canadian Journal of Fisheries and Aquatic Sciences 60:997-1006.

Yamada K, Elith J, McCarthy M, Zerger A (2003) Eliciting and integrating expert knowledge for wildlife habitat modelling. Ecological Modelling 165:251-264. 